\title{
Investigating the radial structure of axisymmetric fluctuations in the TCV tokamak with local and global gyrokinetic GENE simulations
}

\author{
G. Merlo,,${ }^{1} *$ S. Brunner, ${ }^{2}$ Z. Huang, ${ }^{2}$ S. Coda,${ }^{2}$ T. Görler,${ }^{3}$ L. Villard, ${ }^{2}$ A. Bañón \\ Navarro, ${ }^{1}$ J. Dominski, ${ }^{4}$ M. Fontana, ${ }^{2}$ F. Jenko, ${ }^{1,3}$ L. Porte, ${ }^{2}$ and D. Told ${ }^{3}$ \\ ${ }^{1}$ University of California Los Angeles, Los Angeles, California 90095, USA \\ ${ }^{2}$ Ecole Polytechnique Fédérale de Lausanne (EPFL), \\ Swiss Plasma Center (SPC), CH-1015 Lausanne, Switzerland \\ ${ }^{3}$ Max-Planck Institut für Plasmaphysik, Boltzmannstr. 2, D-85748 Garching, Germany \\ ${ }^{4}$ Department of Astrophysical Sciences and Princeton Plasma Physics Laboratory, \\ Princeton University, Princeton, NJ 08544, USA
}

(Dated: November 13, 2017)

\begin{abstract}
Axisymmetric $(\mathrm{n}=0)$ density fluctuations measured in the TCV tokamak are observed to possess a frequency $f_{0}$ which is either varying (radially dispersive oscillations) or a constant over a large fraction of the plasma minor radius (radially global oscillations) as reported in a companion paper [Z. Huang et al, this issue]. Given that $f_{0}$ scales with the sound speed and given the poloidal structure of density fluctuations, these oscillations were interpreted as Geodesic Acoustic Modes, even though $f_{0}$ is in fact smaller than the local linear GAM frequency $f_{\text {GAM. }}$. In this work we employ the Eulerian gyrokinetic code GENE to simulate TCV relevant conditions and investigate the nature and properties of these oscillations, in particular their relation to the safety factor profile. Local and global simulations are carried out and a good qualitative agreement is observed between experiments and simulations. By varying also the plasma temperature and density profiles, we conclude that a variation of the edge safety factor alone is not sufficient to induce a transition from global to radially inhomogeneous oscillations, as was initially suggested by experimental results. This transition appears instead to be the combined result of variations in the different plasma profiles, collisionality and finite machine size effects. Simulations also show that radially global GAM-like oscillations can be observed in all fluxes and fluctuation fields, suggesting that they are the result of a complex nonlinear process involving also finite toroidal mode numbers and not just linear global GAM eigenmodes.
\end{abstract}

\section{INTRODUCTION}

Zonal flows are collective plasma flows of particular interest in fusion research because in certain regimes they are able to regulate turbulence $[1,2]$. In the context of magnetic confinement, zonal flows indicate a plasma flow resulting from a difference between the electrostatic potential on neighbouring poloidal magnetic flux-surfaces. The corresponding radial electric field $\mathbf{E}_{r}$ consequently leads to an $\mathbf{E} \times \mathbf{B}$ rotation of the plasma in the poloidal direction. Zonal flows are non-linearly excited by turbulence, and when turbulent eddies lie radially across these flow layers, they get distorted as a result of the different flow speeds and eventually break into smaller scale ones, resulting in a self-regulating turbulent state [3].

The Geodesic Acoustic Mode (GAM) [4] is a finite frequency Zonal Flow resulting from the coupling of poloidal flows to an axisymmetric pressure perturbation via the geodesic curvature of the magnetic field. They are thus associated with an $\mathrm{n}=0, \mathrm{~m}=0$ electrostatic perturbation, $\mathrm{n}, \mathrm{m}$ being the toroidal and polodial mode numbers, and coupled with a dominant $\mathrm{n}=0, \mathrm{~m}=1$ density fluctuation component. The frequency $f_{\mathrm{GAM}}$ of this mode is predicted to scale proportionally to the ion thermal velocity $v_{t i}=\sqrt{2 T_{i} / m_{i}}$ and inversely proportionally to the major radius $R_{0}$, with a factor of order unity which depends among others on the magnetic safety factor [5], the plasma shape [5,6] and the presence of impurities [7].

Observations of axisymmetric oscillations in the frequency range of the Geodesic Acoustic Mode have been reported by various authors on different machines, see e.g. Refs. [8-12] and references therein. While it is not certain that all these observations are true GAMs, in the following we shall refer to them as "GAMs" for the sake of simplicity.

Thanks to both the very flexible set-up of the diagnostic system and the versatility of the machine in obtaining plasmas with different shapes, which is one of the known parameters affecting the mode frequency, the GAM has also been extensively investigated on the TCV tokamak $[8,13-15]$. The most interesting observation on TCV is the fact 
that the GAM is usually measured as a radially global oscillation, contrary to the predicted dependency of $f_{\mathrm{GAM}}$ on the local sound speed provided by the local linear description of zonal flows. This latter condition, referred to in the following as dispersive mode, has been observed during an edge safety factor ramp-up [14, 16].

In this work, local (flux-tube) and global simulations have been performed investigating TCV relevant conditions, with the goal of reproducing the observed behaviour of axisymmetric fluctuations and in particular the role played by the safety factor profile. The existence of radially global GAM eigenmodes has been analytically predicted [17, 18] and experimentally observed $[9,19-21]$. When the temperature is inhomogeneous, analytic theory predicts the existence of global GAM eigenmodes with frequency $\omega_{\mathrm{GAM}}^{\text {global }}$ and with a finite radial extent. In this case, the mode is predicted to propagate in the low temperature region i.e. in the region where $\omega_{\mathrm{GAM}}^{\text {global }}>\omega_{\mathrm{GAM}}^{\text {local }}$, where $\omega_{\mathrm{GAM}}^{\text {local }}$ is the local GAM frequency. For the TCV cases studied here, the observed global GAM is found, in both experiments and simulations, to evolve at a frequency lower than the local one. Moreover, as will be discussed in the following, in our simulations oscillations at the same frequency as the GAM can also be found in the evolution of the radial profiles of heat and particle fluxes, suggesting a more complex nonlinear coupling between GAM-like axisymmetric oscillations and avalanches [22], and making the distinction between them non trivial.

The remainder of this paper is organized as follows. Section II contains a summary of the experimental results observed when varying the $q$ profile. Section III briefly presents the numerical tool used for carrying out the simulations discussed in this paper. The results obtained modeling actual TCV discharges are described in Secs. IV and V. A discussion is provided in Section VI. Conclusions are drawn in Section VII.

\section{OVERVIEW OF THE TCV EXPERIMENTAL OBSERVATIONS}

Axisymmetric density fluctuations are routinely measured in the outer half of the minor radius of the TCV tokamak $[8,13,16,23]$, in particular thanks to the Phase Contrast Imaging (PCI) diagnostic. Such oscillations are normally observed to possess a "radially global" character, i.e. a global oscillation at constant frequency over a major fraction of the plasma minor radius (typically $\rho_{\psi} \geq 0.65$, where $\rho_{\psi}$ defines the radial coordinate equal to the square root of the normalized poloidal flux), as originally reported for the first time in Ref. [13], and depicted in Figure 1. The
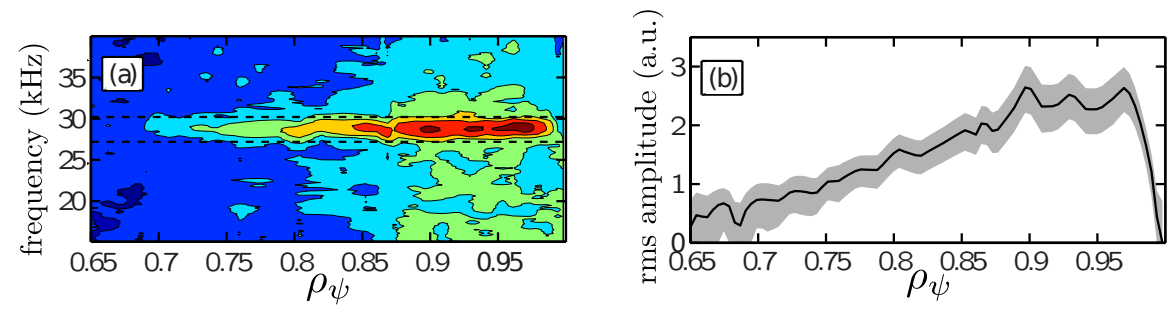

Figure 1: TCV discharge \# 45353. Characterization of the electron density fluctuation in a case showing a radially global nature. Depicted are $(a)$ the auto power spectrum of the density fluctuation measured with the PCI and $(b)$ its RMS amplitude. Adapted from Ref. [13].

frequency of these oscillations is in the range of tens of $\mathrm{kHz}$, and is found to scale proportionally to the sound speed, the latter measured at the location where the mode peaks [8], thus they are interpreted as Geodesic Acoustic Modes (GAMs). This interpretation is furthermore corroborated by the fact that GAM-like coherent oscillations were also observed in the $\mathbf{E} \times \mathbf{B}$ flow (with an $\mathrm{m}=0$ dominant poloidal harmonic), in the electron density (dominated by $\mathrm{m}=1$ ), in the electron temperature and in the poloidal magnetic fluctuation (with a leading $\mathrm{m}=2$ component). Crucially, the latter permits a determination of the perfect axisymmetry of the mode. As already pointed out in Refs. [13, 16], even though the mode frequency scales according to the analytic predictions with respect to e.g. electron temperature and plasma elongation, it is observed to be smaller than expected from these estimates, even at the radial location where the mode amplitude is maximum.

In the latest TCV experimental campaign, several discharges have been performed investigating the behaviour of axisymmetric modes and GAM oscillations, aiming at addressing among others their dependence on the magnetic geometry and on basic plasma parameters as well as differences between limited and diverted plasmas. For a detailed description of the experimental set-ups and the most recent results, the reader is referred to Ref. [16]. The most relevant observation for the current study was the existence of a "radially varying" GAM regime, i.e. with a frequency $f$ varying with radius for large values of the edge safety factor $q_{95}$. Then, when $q_{95}$ was lowered, a transition to a "radially global" mode was observed, as illustrated in Figure 2 of Ref. [16]. 


\section{NUMERICAL SIMULATION TOOL}

All the simulations that we will discuss in this paper have been carried out with the Eulerian gyrokinetic code GENE [24-26]. GENE allows us to perform microturbulence simulations considering gyrokinetic multi-species dynamics, including electrostatic and electromagnetic fluctuations, as well as Intra- and inter-species collisions (both pitch angle and energy scattering). Realistic magnetic geometries can be used via interfaces to MHD equilibrium codes. A detailed description of the code is outside the scope of this paper and the reader is referred to e.g. Ref. [25]. We shall nonetheless briefly recall its essential features.

GENE adopts a field-aligned coordinate system $(x, y, z)$ to represent the fluctuation fields in configuration space. For a tokamak, these coordinates are constructed from the magnetic coordinates $(\psi, \chi, \varphi)$, with $\psi$ being the poloidal magnetic flux, $\chi$ the straight-field-line poloidal angle and $\varphi$ the toroidal angle. One defines the radial coordinate $x$ as a function of the toroidal flux. Throughout all this paper we consider $x=\rho_{\text {tor }} a=\sqrt{\Phi_{\text {tor }} / \pi B_{0}}$, with $\Phi_{\text {tor }}$ the toroidal flux, $B_{0}$ the magnetic field on axis and $a$ the plasma minor radius. The binormal direction is defined by $y=C_{y}(q \chi-\varphi)$, where $q$ is the safety factor and $C_{y}=x_{0} / q\left(x_{0}\right)$ is a constant chosen such that $y$ acquires the units of a length. $x_{0}$ is a reference position corresponding to the location of the flux-tube in local simulations and to the center of the simulation domain in global runs. Finally, $z=\chi$ parametrizes the distance along a field line and is thus usually referred to as the "parallel" direction. Parallel velocity $v_{\|}$and magnetic moment $\mu$ are the velocity space variables. Resolution requirements are different for each specific simulation and will be discussed in the following.

For this work, both the radially local and global (gradient-driven) versions of the code have been used. The binormal direction is in both cases Fourier-transformed, and each $k_{y}$ mode corresponds to a given toroidal mode number $n=k_{y} C_{y}$. In the local (flux-tube) limit of the code, the radial direction $x$ is also Fourier transformed and periodic boundary conditions are applied. In the global version, the $x$-direction is not Fourier transformed and Dirichlet boundary conditions are instead applied on both the perturbed distribution function and the electromagnetic fields. In the latter case radial buffer regions are employed to smoothly damp the fluctuations when approaching the limits of the simulation domain, thereby avoiding numerical instabilities. For all global simulations discussed here, $10 \%$ of the main simulation region is devoted to such buffers at both radial sides. Global simulations also require sources in order to maintain temperature and density profiles. Global gradient-driven runs are performed using a Krook-type operator which acts as a spatially varying source term of both heat and particles. A Krook relaxation rate of the order of a tenth of the maximum linear growth rate is used. For details regarding the heating operator we refer the reader to e.g. Ref. [27]. Finally, all the simulations have been limited to the ion gyroradius scale; numerical fourth order hyperdiffusion is used to avoid spectral pile-ups due to medium $k_{y}$ ETGs.

Two different experimental TCV discharges have been modeled: \#45886, where a "radially coherent GAM" has been measured, and \#49032 where the mode is found to possess a "radially varying" nature. Both discharges are deuterium L-mode limited plasmas. All simulations have been performed assuming heavy electrons $\left(m_{\mathrm{D}} / m_{e}=400\right)$, with the main goal of reducing the overall computational cost, especially of global runs, and allowing us to investigate different experimental conditions. The focus of this paper is indeed on qualitatively reproducing the experimental observations regarding the spatial nature of axisymmetric fluctuations. We have in particular investigated the effect of finite collisionaly, a parameter know to have a strong impact on turbulence in TCV relevant conditions [28] and in general affecting the dynamics of zonal flows, and of the safety factor profile. A quantitative investigation, aiming at reproducing both the experimental transport and fluctuation level, will likely require not only to adopt the realistic deuterium-to-electron mass ratio but also to include impurities which are known to potentially strongly affect the turbulence level [28] as well as the linear GAM frequency [7]. These significantly more expensive investigations are left for future work.

\section{EXPERIMENTAL CONDITIONS FOR "RADIALLY COHERENT GAM"}

\section{A. TCV experimental discharge}

In order to investigate the experimental TCV conditions corresponding to the "radially coherent GAM", we consider discharge \# 45886. This L-mode limited plasma corresponds to a power ramp-up, with the external EC heating increased from 0.4 MW to 0.9 MW. For our investigation we focus on time $\mathrm{t}=1.1 \mathrm{~s}$, when $0.6 \mathrm{MW}$ external heating power is applied. The radial profiles of electron density and temperature (for main ions and electrons) are depicted in Figure 2, together with the safety factor profile $q$. Impurities are not accounted for, thus their concentration profile is not shown. We remark that the effective charge, defined as $Z_{\text {eff }}=\sum_{i} n_{i} Z_{i}^{2} / n_{e}$ summed over all ion species, takes on the value $Z_{\text {eff }}=3.4$, with carbon $\left(Z_{C}=6\right)$ the dominant impurity (the corresponding ratio of carbon to main ion concentration is $\left.n_{\mathrm{C}} / n_{\mathrm{D}}=15 \%\right)$. A coherent oscillation is in this case measured at $28 \mathrm{KHz}$, as shown in Figure 1 . 

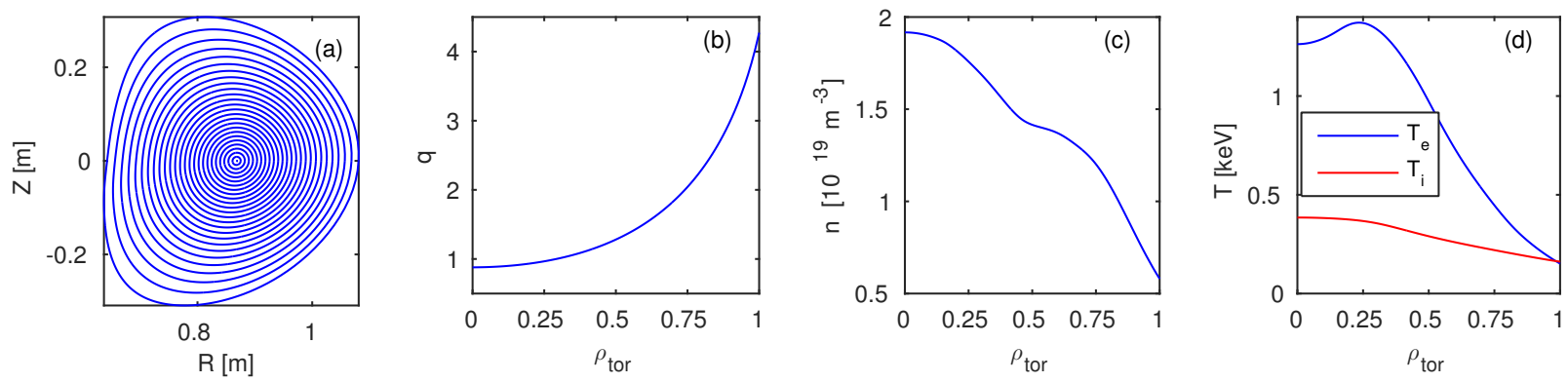

Figure 2: (a) Reconstructed magnetic equilibrium (shown are contours of constant poloidal flux), (b) safety factor $q$ profile, (c) electron density $n_{e}$ and $(d)$ electron and ion temperature profiles as a function of $\rho_{t o r}\left(T_{e}\right.$, in blue, and $T_{i}$, in red, respectively). Profiles measured for TCV discharge \# 45886 at time t=1.1s presenting "radially coherent GAM".

\section{B. Local simulation results}

We first carry out a series of flux-tube simulations at different radial locations, in order to investigate the local properties of turbulence and the behaviour of axisymmetric fluctuations. We look at $\rho_{\text {tor }}=0.6,0.7$ and 0.85 . The experimental magnetic equilibrium, reconstructed with the CHEASE code [29], has been used as well as the measured plasma profiles. The plasma parameters used for the simulations are detailed in Table I.

\begin{tabular}{lccc}
\hline \hline & $\rho_{\text {tor }}=0.6$ & $\rho_{\text {tor }}=0.7$ & $\rho_{\text {tor }}=0.85$ \\
\hline \hline$R_{0} / L_{T_{e}}$ & 9.32 & 10.47 & 12.82 \\
$R_{0} / L_{T_{i}}$ & 3.40 & 3.48 & 3.85 \\
$R_{0} / L_{n}$ & 1.48 & 2.92 & 8.62 \\
$T_{i} / T_{e}$ & 0.35 & 0.44 & 0.65 \\
$q_{0}$ & 1.51 & 1.83 & 2.61 \\
$\hat{s}$ & 1.07 & 1.46 & 2.27 \\
$\beta$ & $0.1910^{-2}$ & $0.1310^{-2}$ & $0.5610^{-3}$ \\
$\nu_{e i}\left[R_{0} / c_{s}\right]$ & 0.064 & 0.110 & 0.261 \\
\hline \hline
\end{tabular}

Table I: Local parameters used for carrying out flux-tube simulations investigating the "radially coherent GAM" conditions of TCV discharge \# 45886.

Normalized logarithmic gradients $R_{0} / L(n, T)$ are defined according to the following relation:

$$
R_{0} / L(\mathrm{n}, \mathrm{T})=-\mathrm{R}_{0} / \mathrm{ad} \log (\mathrm{n}, \mathrm{T}) / \mathrm{d} \rho_{\text {tor }}
$$

where $R_{0}$ is the major radius of the machine, $R_{0}=0.88 \mathrm{~m}$, and $a=\sqrt{\Phi_{\mathrm{LCFS}} / \pi B_{0}}$ provides a measure of the plasma minor radius. $q_{0}$ is the local value of the safety factor and $\hat{s}=x / q d q / d x$ the local magnetic shear. Electromagnetic effects are accounted for using the experimental value of $\beta_{e}=2 \mu_{0} p_{e} / B_{0}^{2}$; here $p_{e}$ is the local electron pressure. For the simulations in which finite collisionality is accounted for, it is evaluated based on the experimental values . Inter- and intra-species collisions are retained and modelled with a linearized Landau collision operator. All collision frequencies are consistently derived from the values of $\nu_{e i}$ and local values of temperature and density. In particular, $\nu_{e i}=3 \sqrt{\pi} / 4 \tau_{e, i}$, where $\tau_{e, i}$ is the ion-electron collision time $\tau_{e, i}=3(2 \pi)^{3 / 2} \epsilon_{0}^{2} T_{e}^{3 / 2} m_{e}^{1 / 2} / n_{i} Z_{i}^{4} e^{2} \log \Lambda, m_{e}$ is the electron mass and $\log \Lambda$ is the Coulomb logarithm.

Two sets of linear runs have been performed, switching collisionality on and off. In both scenarios, for the parameters being considered here, the dominant linear instabilities are Trapped Electron Modes (TEM) which, as can be seen in Figure 3 , are only weakly stabilized by the inclusion of collisions. This stabilization mainly affects the low $k_{y}$ modes and is weaker as one considers more radially outward positions. As an example, the growth-rate of the $k_{y} \rho_{s}=0.3$ mode is lowered by $\sim 50 \%$ at $\rho_{\text {tor }}=0.6$, by $\sim 20 \%$ at $\rho_{\text {tor }}=0.7$ and only $\sim 5 \%$ at $\rho_{\text {tor }}=0.85$. At higher wave numbers Electron Temperature Gradient (ETG) modes are unstable. Their contribution will be neglected in all nonlinear simulations which are limited to the ion scale. Growth rates are mostly affected by the temperature gradient while changing $R / L_{n}$ by $\pm 30 \%$, a variation idicative of the experimental error bars, is not found to significantly affect them.

Nonlinear simulations have been performed at each of the three aforementioned locations; a typical grid size for these runs is $n_{k_{x}} \times n_{k_{y}} \times n_{z} \times n_{v_{\|}} \times n_{\mu}=256 \times 64 \times 36 \times 64 \times 16$. The minimum $k_{y}$ mode retained is adapted 

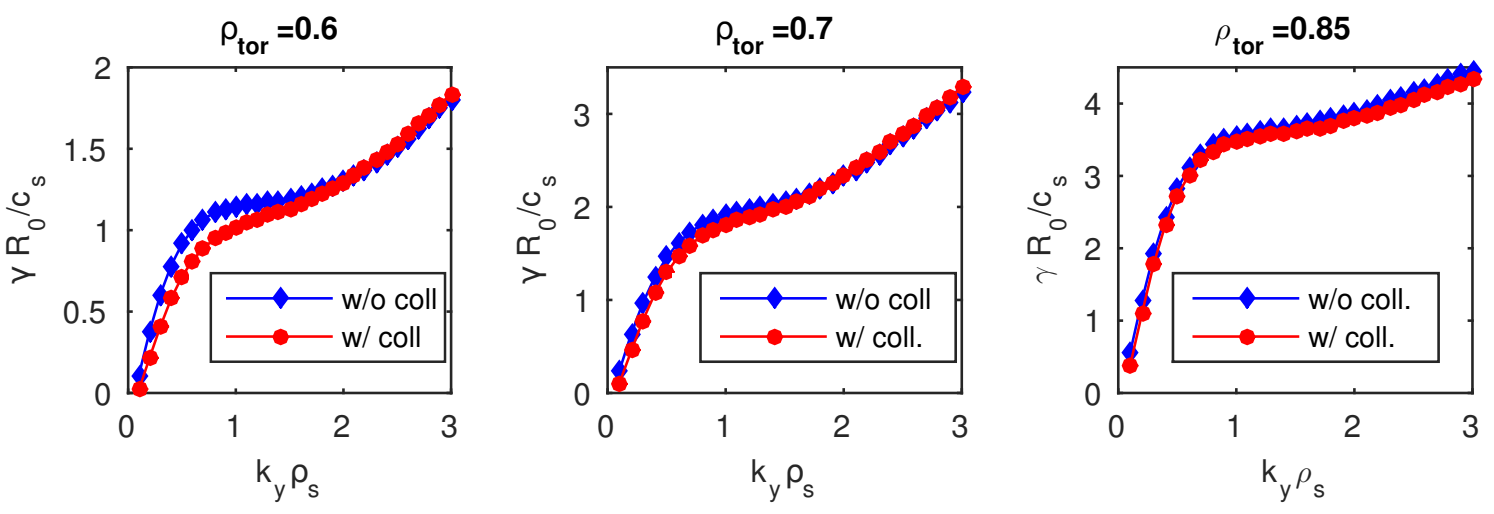

Figure 3: Growth rates $\gamma$ of the most unstable mode as a function of the wave number $k_{y} \rho_{s}$ evaluated at the radial locations $\rho_{\text {tor }}=0.6,0.7$ and 0.85 . All modes possess a negative real frequency, i.e. they propagate in the electron diamagnetic direction. Results obtained neglecting (resp. retaining) finite collisionality are depicted in blue (resp. red).

such that it corresponds at each location to the toroidal mode number $n=2$, thus simulating a toroidal wedge of half-torus and matching the one used for global runs. The velocity space grids of each $j-$ species are set from -3 to 3 for $v_{\|} / v_{t h, j}$ and from 0 to 9 for $\mu B_{0} / T_{j}, v_{t h, j}=\sqrt{2 T_{j} / m_{j}}$ being the thermal velocity and $B_{0}$ the magnetic field on axis. Even though it is not the aim of this paper to reproduce the experimental transport level, the simulated
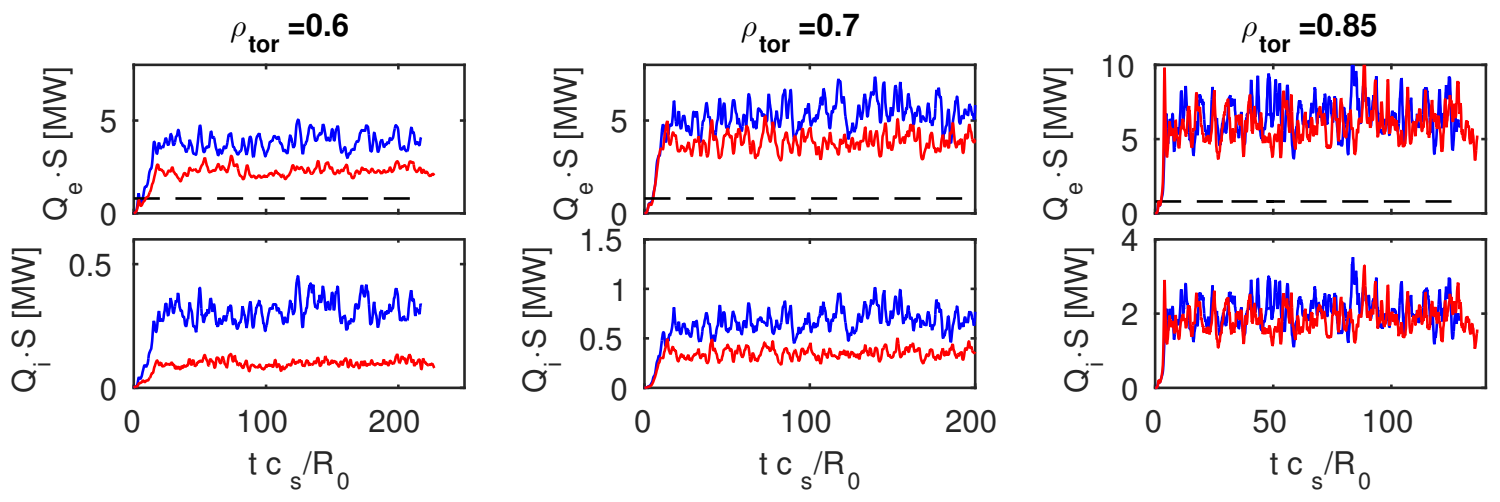

Figure 4: Time traces of the simulated heat fluxes $Q_{e}$ of electrons (upper plots) and $Q_{i}$ of ions (lower plots). From left to right shown are the results obtained considering the radial locations $\rho_{\text {tor }}=0.6,0.7$ and 0.85 . When plotting the heat flux (in red for collisional and in blue for collisionless simulations), only the electrostatic component is shown, as the corresponding electromagnetic part is smaller by at least an order of magnitude in all cases. The experimental electron heat flux, determined via power balance, is shown with dashed black lines.

time traces of nonlinear heat powers $\mathrm{Q} \cdot \mathrm{S}$ in MW obtained at the different radial locations are depicted in Figure 4 together with the experimental value of $0.8 \mathrm{MW}$ estimated via power balance analysis. The heat flux $Q$ is evaluated from simulations as $Q=\langle\mathbf{Q} \cdot \nabla x\rangle_{S}$, where $\langle\cdot\rangle_{S}$ indicates the surface average $\langle\cdot\rangle_{S}=(1 / S) \int_{s} \cdot \mathrm{d} \Sigma$, with $S=\int \mathrm{d} \Sigma$ the area of the flux-surface of interest. Here, $\mathbf{Q}$ is the heat flux, defined as $\mathbf{Q}=\int\left(1 / 2 m v^{2} \delta f v_{\mathbf{E} \times \mathbf{B}}\right) d^{3} v$, where $\delta f$ is the fluctuating part of the distribution function and $v_{\mathbf{E} \times \mathbf{B}}$ the $\mathbf{E} \times \mathbf{B}$ velocity. Concerning the experimental power balance, it is shown only for the electron channel, since in these TCV discharges there was no external ion heating and the equipartition power can be neglected. Furthermore, when plotting simulation results, we only show the electrostatic component as the electromagnetic counterpart is smaller by at least one order of magnitude at all radii. As expected, transport is dominated by the electron channel with fluxes significantly larger than the experimental heating powers. The difference between simulation and experiments is increasing from core to edge: at $\rho_{\text {tor }}=0.6$ the simulated electron heat flux is $\sim 4 \mathrm{MW}$, i.e. five times the experimental heating power, while at $\rho_{\text {tor }}=0.85$ the ratio simulated-to-experiments increases to almost 8. Similarly, the ion heat flux increases significantly as well, approximately by a factor of 10 in the collisional case, when comparing the innermost to the outermost position being simulated. Besides invoking finite machine size stabilization effects [30], this discrepancy is largely due to the heavy electron assumption. Preliminary simulations (not shown here) reveal that using the experimental mass ratio $m_{\mathrm{D}} / m_{e}=3670$ lowers the nonlinear fluxes by more than a factor of two, bringing simulations closer to experiments. 
In addition, including impurity is also expected to help matching the measured transport level due to the increased collisionality which stabilizes TEMs and to the dilution of the main ions.

With the aim of investigating the behaviour of axisymmetric fluctuations, we study the spatial and temporal behaviour of the poloidal $\mathbf{E} \times \mathbf{B}$ velocity, associated to the flux-surface-averaged electrostatic potential defined as

$$
v_{\mathbf{E} \times \mathbf{B}}=\frac{1}{B_{0}} \frac{\partial\langle\phi\rangle_{\mathrm{FS}}}{\partial x},
$$

where $\langle\cdot\rangle_{\mathrm{FS}}$ stands for the flux surface average $\langle\cdot\rangle_{\mathrm{FS}}=\int \cdot J^{x y z} d y d z / \int J^{x y z} d y d z, J^{x y z}$ being the Jacobian of the $(x, y, z)$ coordinate system. As an example, the results relative to the collisionless simulation at $\rho_{\text {tor }}=0.7$ is depicted in Figure 5 .

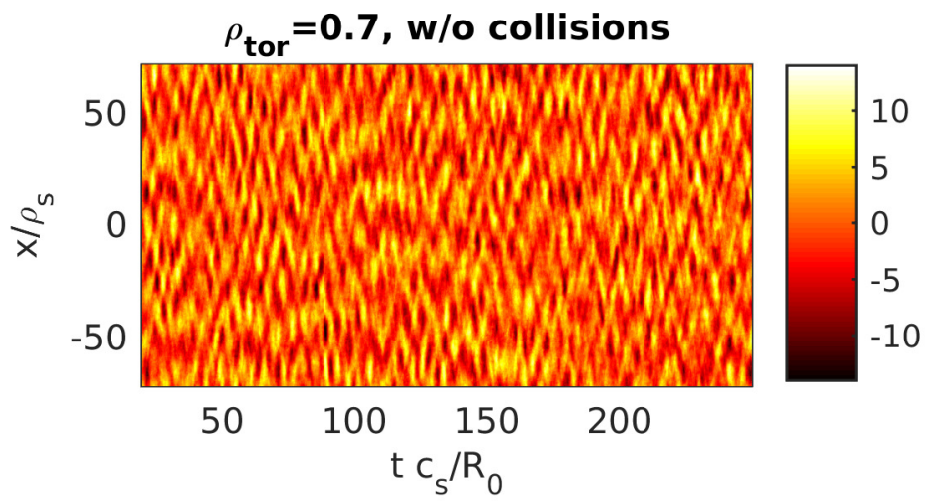

Figure 5: Simulated flux-surface-averaged $\mathbf{E} \times \mathbf{B}$ velocity as a function of radial coordinate $x$ and time $t$. Simulation carried out assuming TCV conditions for the "radially coherent GAM" at $\rho_{\text {tor }}=0.7$ and neglecting collisions.

In order to isolate any dominant frequency, we evaluate the power spectral density of the simulated signals at each radial position using Welch's algorithm [31]. The time traces are divided into overlapping intervals approximately $\sim 40 R_{0} / c_{s}$ long and a Hamming window is applied to each interval. We have verified that varying the length of the intervals by $\pm 20 R_{0} / c_{s}$ does not significantly affect the results. The results obtained in this way are shown in Figure 6. We furthermore plot in Figure 7 the corresponding radially averaged quantities, to facilitate the evaluation of the leading frequency and the comparison between collisional and collisionless results. The linear GAM frequency, estimated by carrying out the Rosenbluth-Hinton test [32], is shown for completeness with a dashed white line.

Depending on the position being analyzed, we observe a different behavior of the $\mathbf{E} \times \mathbf{B}$ velocity and a different effect of collisions. Considering the collisionless results first, at the innermost position considered $\left(\rho_{\text {tor }}=0.6\right)$ there is not a clear frequency dominating the spectra of $v_{\mathbf{E} \times \mathbf{B}}$, which shows a broad contribution from all modes with a frequency below $\sim 60 \mathrm{kHz}$. At $\rho_{\text {tor }}=0.75$ and 0.8 we instead observe a clear peak at a finite frequency, of approximately 32 $\mathrm{kHz}$ for both positions. The inclusion of collisions is found to significantly affect the results, and consistently with linear growth rates and nonlinear flux results, the effect is strongest at the innermost positions. At $\rho_{\text {tor }}=0.6$ retaining collisionality is found to strongly damp the contribution from all finite frequencies. A "knee" remains at around $\sim 50 \mathrm{kHz}$ but it is too weak to be clearly identified as a finite frequency oscillation. At $\rho_{\text {tor }}=0.7$ collisions are observed to up-shift the dominant frequency, by almost $25 \%$, from $\sim 33 \mathrm{kHz}$ to $\sim 40 \mathrm{kHz}$. We note that in fact a peak at $\sim 40 \mathrm{kHz}$ was already present in the collisionless spectra, but much weaker than the dominant, lower frequency one. Finally, at $\rho_{\text {tor }}=0.85$ the effect of collisionality is weak, visible only as a damping of the amplitudes of the low-frequency components. The dominant nonlinear frequencies are summarized in Table II. These results are not

\begin{tabular}{cccc}
\hline \hline & $\rho_{\text {tor }}=0.6$ & $\rho_{\text {tor }}=0.7$ & $\rho_{\text {tor }}=0.85$ \\
\hline \hline $\mathrm{f}_{\text {lin, GAM }}[\mathrm{kHz}]$ & 60.6 & 49.9 & 39.56 \\
$\mathrm{f}[\mathrm{kHz}], \mathrm{w} / \mathrm{o}$ coll. & - & 32.8 & 31.7 \\
$\mathrm{f}[\mathrm{kHz}], \mathrm{w} /$ coll. & - & 40.2 & 31.1 \\
\hline \hline
\end{tabular}

Table II: Dominant frequency, in $\mathrm{kHz}$, of axisymmetric oscillations obtained from analyzing the $\mathbf{E} \times \mathbf{B}$ velocity in nonlinear local simulations. Results at $\rho_{\text {tor }}=0.6$ are not listed as a clear peak is not found. The linear estimate obtained from the Rosenbluth-Hinton test is listed as well as $\mathrm{f}_{\text {lin,GAM }}$.

obvious to interpret and compare to the experiments, especially in relation to the radially global structure of the 

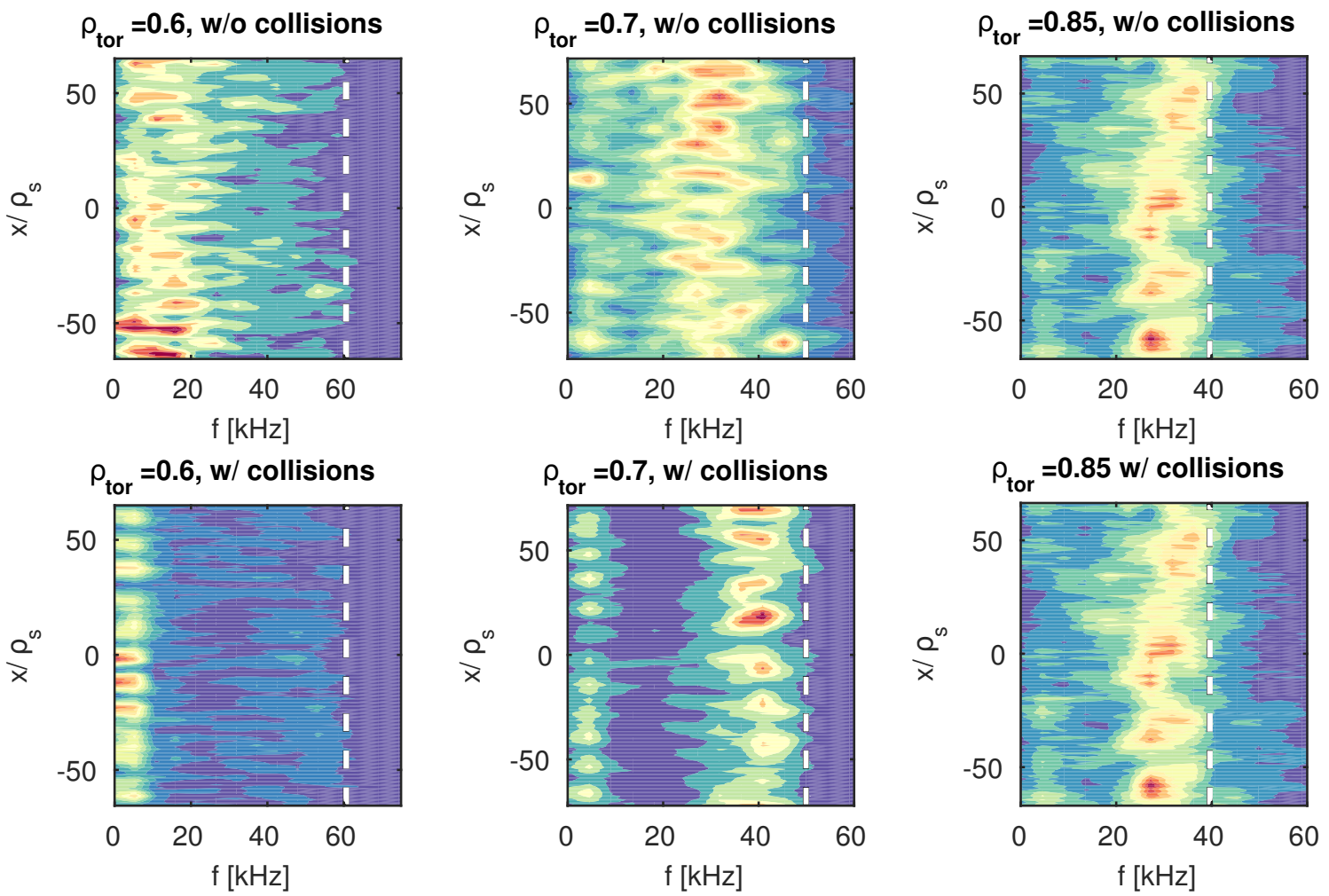

Figure 6: Power spectra densities of the simulated $\mathbf{E} \times \mathbf{B}$ velocity as a function of radius and frequency. Shown from left to right are the results obtained at $\rho_{\text {tor }}=0.6,0.7$ and 0.85 . In each column the upper plot presents collisionless results while the lower plot shows collisional results. The color coding is the same for each position. The linear GAM frequency measured performing the Rosenbluth-Hinton test is shown with a white dashed line. Simulations consider TCV conditions for the "radially coherent GAM".
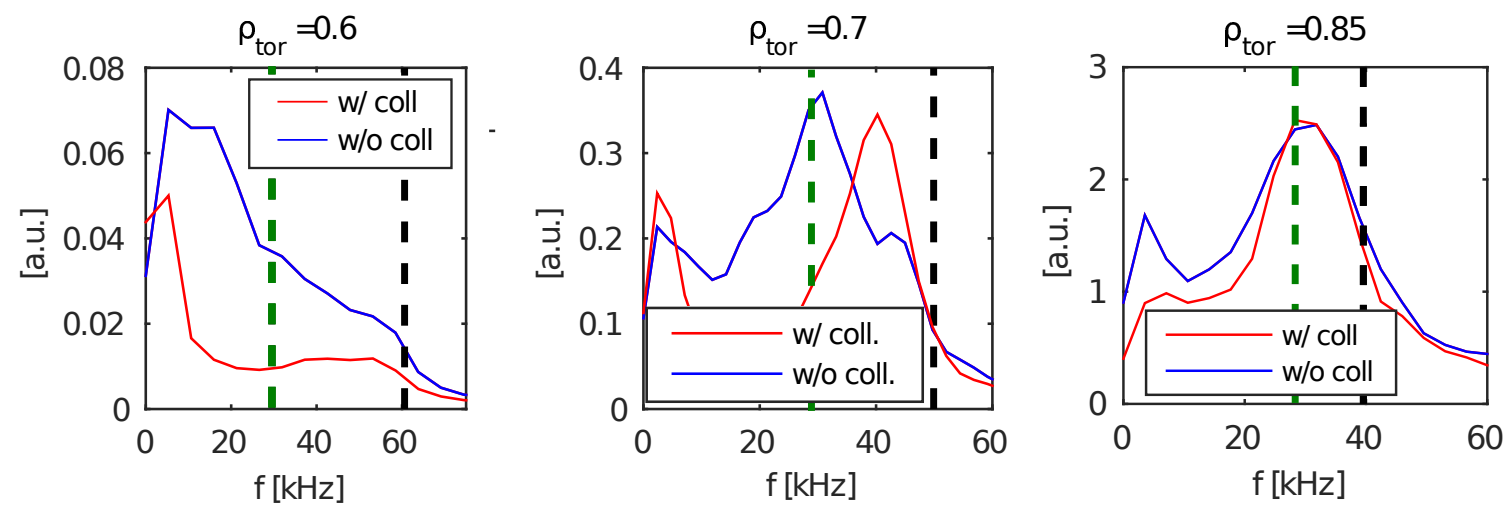

Figure 7: Radially averaged power spectra of the $\mathbf{E} \times \mathbf{B}$ velocity as a function of frequency for the same flux-tube simulations as in Fig. 6. Collisionless (resp. collisional) results are shown in blue (resp. red) while the linear GAM frequency is plotted with a black dashed line. The experimentally measured frequency $(28 \mathrm{kHz})$ is shown with a vertical green dashed line. Simulations consider TCV conditions of the "radially coherent GAM".

observed fluctuations. For instance, the collisionless results seem to indicate that the two locations $\rho_{\text {tor }}=0.7$ ad 0.85 are characterized by nearly the same frequency, which would agree with a global mode as experimentally observed. The collisional results on the other hand provide a very different result, with frequencies varying as a function of the radial position. Global simulations are therefore necessary in order to understand how these modes coexist and interact with each other. We also remark that zonal flows seem to be playing a major role in saturating the turbulence. Collisional simulations where the zonal flows are artificially removed (not shown here) show an increase by up to one order of magnitude of the transport level. This appears to be in contrast with what described in Refs. [33] and [34], 
where zonal flows are found to be irrelevant for TEM cases where $\eta_{e} \gg 1$, where $\eta_{e}=\mathrm{d} \ln T_{e} / \mathrm{d} \ln n$. Almost all plasma parameters of our case are however different, thus the comparison is not obvious and requires a more detailed investigation, which we leave for future work.

\section{Global simulation results}

We discuss below the results obtained with global simulations, still modeling the "radially coherent GAM" scenario. In this case, the simulation radial domain has been centered at $\rho_{\text {tor }}=0.7$ and covers the annulus $0.42<\rho_{\text {tor }}<0.98$. For the profiles being simulated (see Fig. 2) the finite machine size parameter $\rho^{*}=\rho_{s} / a$ takes the value $\rho^{*}=\sim 1 / 122$ at the center of the simulation domain. The radial domain thus corresponds to $L_{x} \sim 69 \rho_{s}$ and it is discretized using 256 radial grid points. As already mentioned, $10 \%$ of the simulation domain on both radial sides is devoted to numerical buffer regions, which will be ignored in all the following plots. We consider 48 toroidal mode numbers (the minimum toroidal mode number retained is set to $n_{\min }=2$ ), while $32 n_{z}$ points are used to represent the parallel direction $z$. Finally, we consider the velocity space domain $L_{v_{\|}} \times L_{\mu}=4.14 \times 18$ (normalized with respect to the reference values at the center of the simulation domain), discretized with $n_{v_{\|}} \times n_{\mu}=110 \times 48$ points.

Similarly to the flux tube results, a pair of simulations, with and without collisions, has been carried out. Both gradient driven simulations are run up to $\sim 230 R_{0} / c_{s}$, in order to collect sufficient statistics. The simulated heat
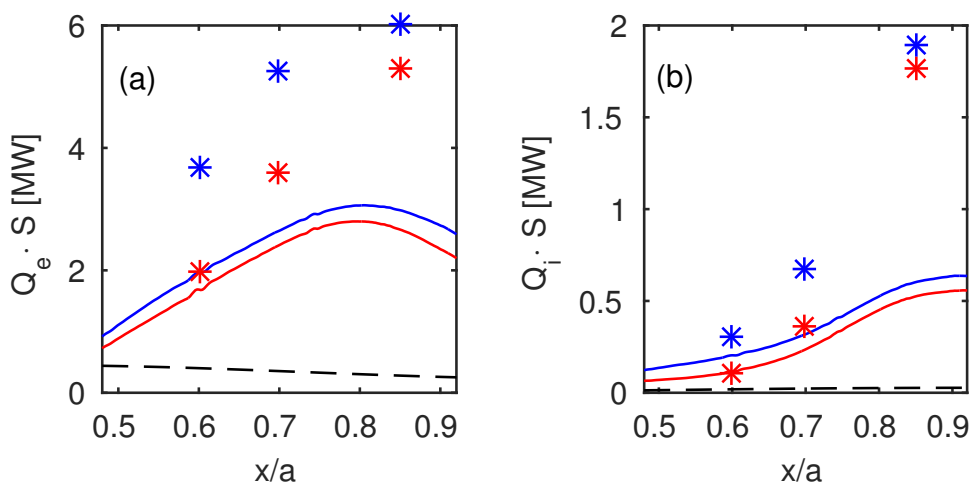

Figure 8: Global simulations results for the "radially coherent GAM" case. Heat fluxes in MW as a function of the minor radius for $(a)$ the electron and $(b)$ the ion channel. Collisional results are depicted in red, while collisionless in blue. Local results are reported with stars following the same color coding, while the experimental heating power is indicated with a black dashed line.

transfer rates as a function of the minor radius are shown in Figure 8 (red solid lines for collisional results and blue collisionless, while the black dashed line indicates the experimental value). For completeness the flux tube results are also plotted with colored stars using the same color coding to distinguish between collisional and collisionless simulations. We first point out the strong difference between local and global simulation results. The latter provide at all positions a flux which is significantly lower than the estimates obtained with flux-tube runs, confirming, as expected from the small machine size, the necessity of carrying out global simulation in order to reproduce the actual transport level of TCV. A different overestimation by the local results can nonetheless be observed depending on which channel and position one considers. For the transport through the electron channel, one observes that at $\rho_{\text {tor }}=0.6$ and 0.7 the overestimation is approximately twofold, while at $\rho_{t o r}=0.85$ local predictions are larger by almost a factor of 2.5 . For the ion channel similar local-to-global ratios are observed at the two innermost radii, but at the outermost position the difference increases, with flux-tube transport estimation more than four time larger than the global ones. We also note a lower effect of collisionality in global simulation compare to local ones. Global simulations results remain in any case significantly higher than the experimental value, and this, as already discussed before is understood mainly as a consequence of the heavy electrons assumption. We also remark that the simulated heat flux is increasing with radius (peaking at $\rho_{\text {tor }} \sim 0.8$ for the electron channel and close to the outermost simulated radial location for the ions), which is in disagreement with the results obtained from the experimental power balance.

The time-averaged effective temperature and density profiles are shown in Figure 9. We observe a small relaxation of the profiles from the imposed background, larger for the electron temperature compared to the ion. This relaxation is however small, less than $5 \%$, and therefore is not expected to affect the results. The same relaxation is found for collisional and collisionless runs, with the corresponding profiles in Figure 9 essentially overlapping each other. 

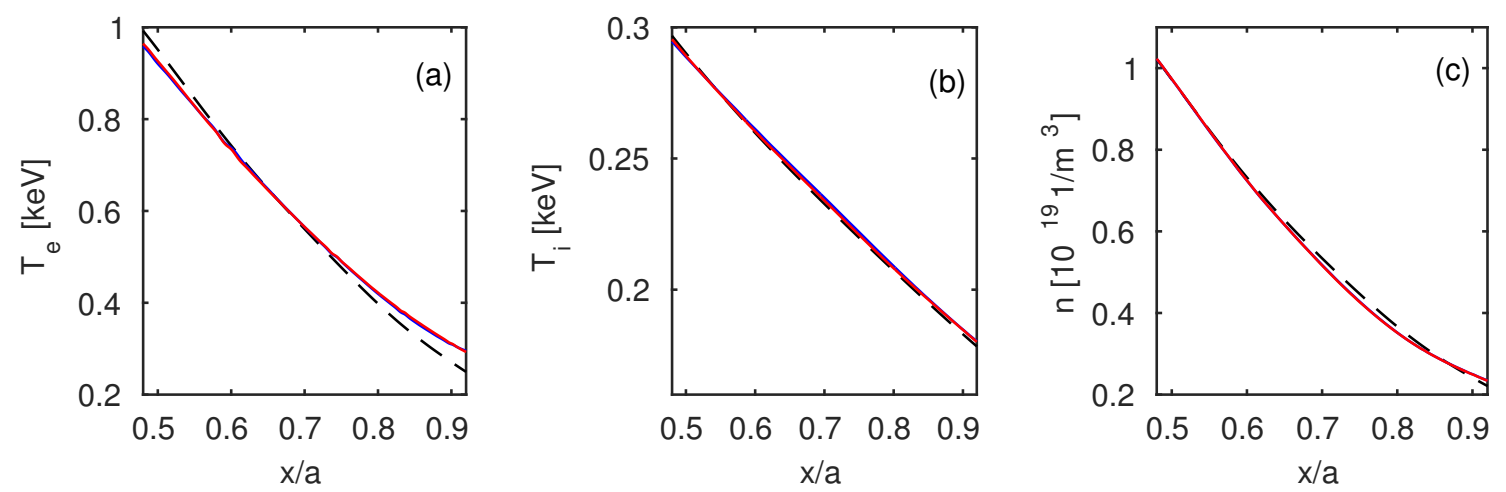

Figure 9: Same simulations as in Figure 8. Time averaged profiles of $(a)$ electron temperature $T_{e},(b)$ ion temperature $T_{i}$ and (c) density $n$. Results from collisional simulations are shown in red, collisionless in blue, while the background profiles are shown with black dashed lines.

We have repeated the same analysis of the axisymmetric modes by inspecting the associated $\mathbf{E} \times \mathbf{B}$ velocity, whose contours as function of the radial variable and time are shown in Figure 10. In both the collisionless and collisional

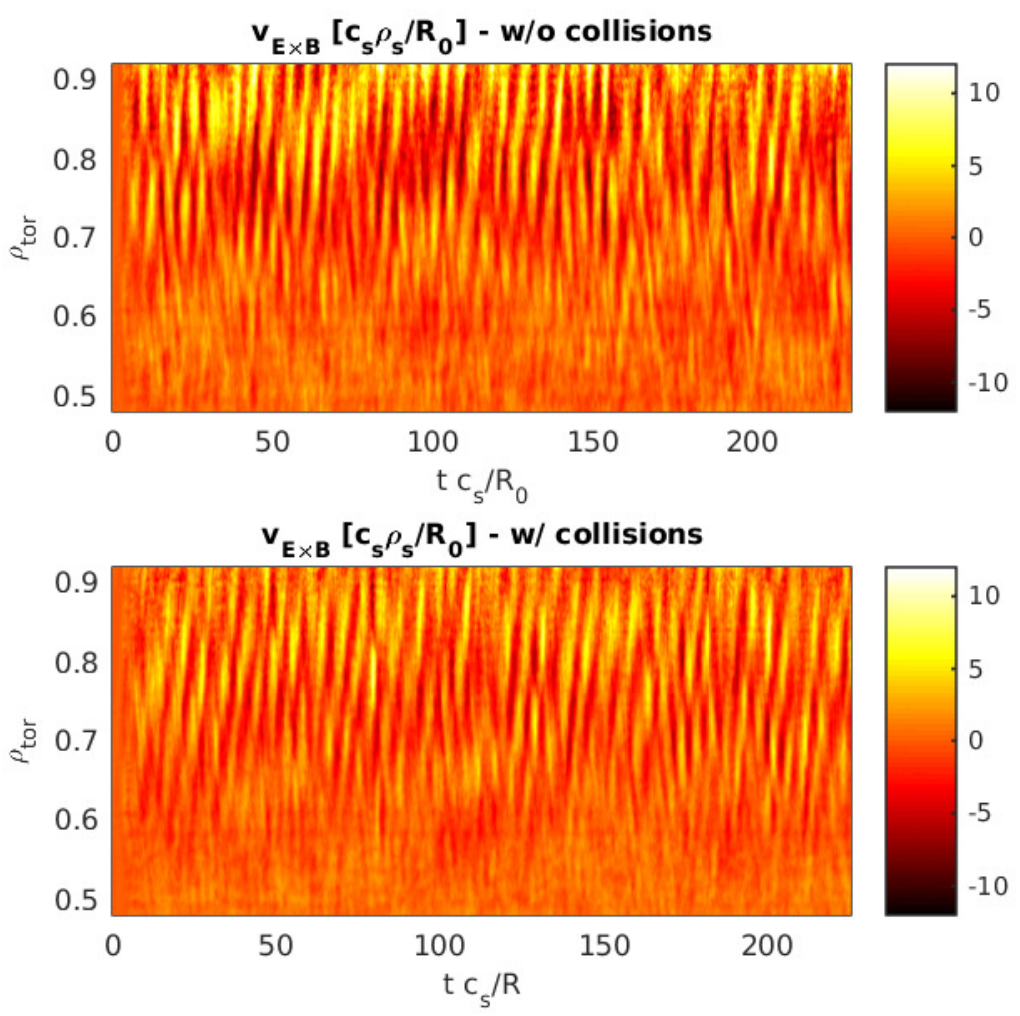

Figure 10: Same simulations as in Figure 8. Simulated $\mathbf{E} \times \mathbf{B}$ velocity as a function of radial coordinate $\rho_{t o r}$ and time $t$. The upper plots shows collisionless results, the lower collisional.

simulations one observes the presence of mainly outward propagating structures, dominantly in the outer part of the plasma, i.e. from $\rho_{\text {tor }} \sim 0.65$ up to the upper end of the simulation domain. To characterize the frequency of these oscillations, we performed a Fourier time analysis in the same way as already done for local simulations. The results are depicted in Figure 11 where the power spectra of $v_{\mathbf{E} \times \mathbf{B}}$ are compared to the local nonlinear results and the local linear GAM frequency $f_{\text {lin,GAM }}$. The latter is obtained by carrying out the Rosenbluth-Hinton test for conditions relative to different radial positions and from applying analytic estimates. In particular, we use the dispersion relation 
provided in Ref. [5], which accounts for plasma shaping, elongation $\kappa$ and inverse aspect ratio $\epsilon=\rho / R_{0}$ :

$$
f_{\text {lin }, \mathrm{GAM}}=\frac{v_{t i}}{2 \pi R_{0}} \frac{\sqrt{7+4 \tau}}{2} \sqrt{\frac{2}{\kappa^{2}+1}}\left(1-\epsilon^{2} \frac{9 \kappa^{2}+3}{8 \kappa^{2}+8}\right)\left[1+\frac{\kappa^{2}+1}{4 q^{2}}\left(\frac{23}{8}+2 \tau+\frac{1}{2} \tau^{2}\right)\left(\frac{7}{4}+\tau\right)^{-2}\right]
$$

where $\tau=T_{e} / T_{i}$ and, for simplicity, we have neglected all other plasma shaping factors (e.g. the Shafranov shift $\Delta$ and the radial derivative of elongation $\kappa^{\prime}$ ) which lead to minor corrections in our cases. Finite radial wavelength effects have also been neglected.
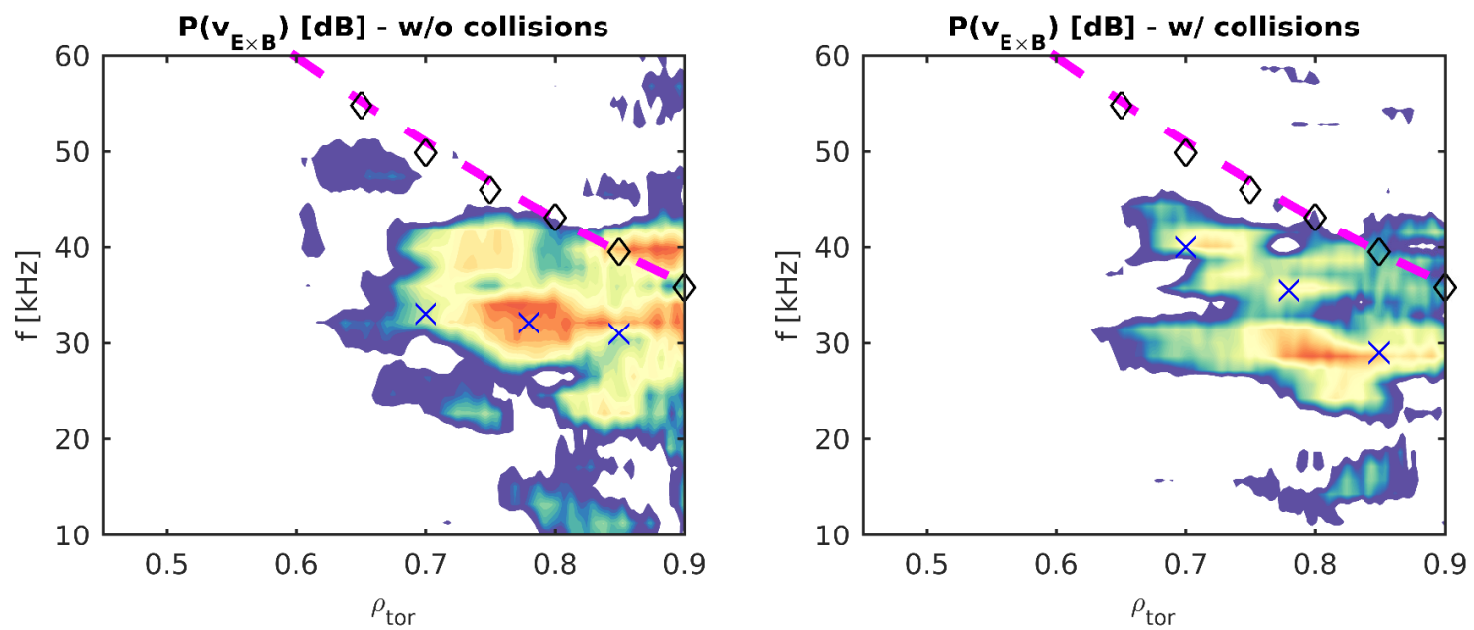

Figure 11: Same simulations as in Figure 8. Power spectra density of the $\mathbf{E} \times \mathbf{B}$ velocity for the (left) collisionless and (right) collisional simulations, $\mathrm{dB}$ scale with the same color coding for the two cases. The magenta dashed line indicates the analytic linear GAM frequency estimate (Eq. (3), obtained from Ref. [5]), while black diamonds correspond to the frequency simulated in flux-tube via the Rosenbluth-Hinton test. Nonlinear local results discussed in the previous section are reported with blue crosses.

One notes the almost perfect agreement between GENE local linear results (black diamonds) and the analytic estimates provided by Eq. (3) (magenta dashed lines). Both local and global nonlinear numerical results are, however, lower than the linear estimates, in agreement with the experimental measurements. The nonlinear global spectra, in particular the one obtained for the collisionless case, appear to be dominated by a single frequency oscillation, around $\sim 30 \mathrm{kHz}$ and covering the radial domain $\rho_{\text {tor }} \geq 0.65$. This dominant frequency is somewhat lower in the collisional simulation, $28.6 \mathrm{kHz}$, compared to the collisionless case, where $\sim 32 \mathrm{kHz}$. We also note that when collisions are neglected, the dominant mode appears to be broader in frequency compared to the results obtained with collisions active.

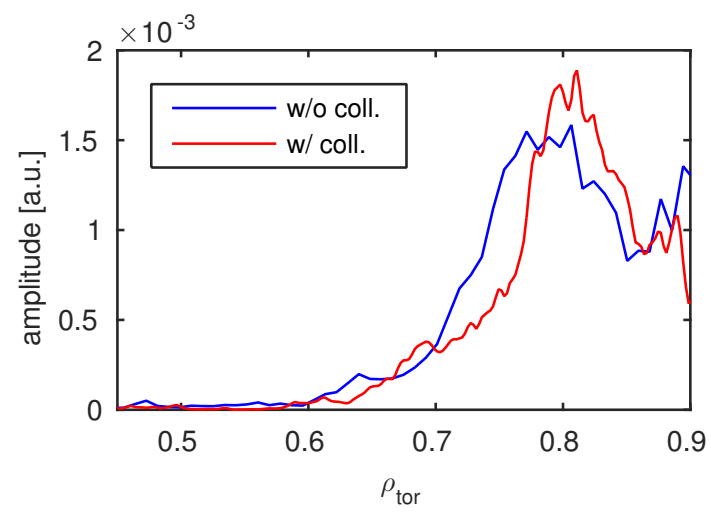

Figure 12: Spectral power density of the simulated $v_{\mathbf{E} \times \mathbf{B}}$ taken at the dominant frequency as a function of the radial location. Collisionless results are shown in blue and for the frequency $f=32 \mathrm{kHz}$, while collisional in red and taken at $28.6 \mathrm{kHz}$.

In both cases, the amplitude of the leading mode increases with radius, reaches a peak at $\rho_{\text {tor }} \simeq 0.8$ and then decreases towards the LCFS, as shown in Figure 12. We observe the collisional case to be more radially strongly 
peaked and outward shifted with respect to the collisionless case. Finally, other modes at different frequencies are also present. Clearly visible in both simulations is a component at approximately $40 \mathrm{kHz}$ at $\rho_{\text {tor }} \simeq 0.7$, which in the collisionless run can be observed also at $\rho_{\text {tor }} \simeq 0.85$. A lower frequency oscillation, at $\simeq 25 \mathrm{kHz}$, is also found, stronger in the collisionless case.

Comparing global with local results discussed in the previous section (reported in Figure 11 with blue crosses), one observes that at the outermost position considered with local simulations, $\rho_{\text {tor }}=0.85$, a good agreement is obtained between local and global results, with a difference $\simeq 10 \%$. At $\rho_{t o r}=0.7$ the comparison is instead more complex. It appears that local simulations "select" among the different frequencies that contribute to the global spectra at that particular position the one with largest amplitude. In order to further clarify this point, we have carried out one extra local simulation considering the plasma parameters of $\rho_{t o r}=0.78$. We observe that in the collisionless simulation the local nonlinear frequency remains approximately $32 \mathrm{kHz}$, whereas in the collisional case the results from the flux-tube run show a higher frequency, $\sim 35 \mathrm{kHz}$. While this latter frequency corresponds to a local peak in the corresponding global results, it is not the frequency with the largest amplitude at this radial position. It therefore appears that local nonlinear simulations provide a local nonlinear frequency estimate, smaller than the corresponding linear local one, but not necessarily related to the largest magnitude that can be obtained at that radial location from global simulations. The apparent agreement between local and global results for the collisionless case can thus also be partly coincidental, and our results indicate how local simulations are, as expected, not suited for reproducing features that possess a "radially global" nature, as the one observed in TCV. Moreover, they are more sensitive in terms of the result they provide, to the physical model used, in particular in this case to the effect of collisions.

As already mentioned, all these $\mathbf{E} \times \mathbf{B}$ oscillations are found at a frequency well below the local linear GAM one, and the dominant nonlinear frequency appears to approach the corresponding local linear estimate only close to the LCFS. This is in disagreement with the prediction of the existence of global linear GAM oscillations with finite radial extent due to the presence of temperature gradients, see e.g. Ref. [2]. These modes are expected to propagate in regions where their frequency is larger than the local GAM one, whereas we clearly observe in our simulation the opposite behaviour with an outward propagation at $f_{\text {GAM }}<f_{\text {lin, GAM }}$

Finally, we also remark a very good agreement of the dominant axisymmetric mode frequency with the experimental observations. Despite the strongly simplified model, the heavy electron assumption and neglecting impurities, the simulated mode frequency agrees within $10 \%$ with the measurements.

\section{EXPERIMENTAL CONDITIONS FOR "RADIALLY-VARYING GAM"}

\section{A. Plasma experimental profiles}

The same analysis carried out considering the experimental conditions for the "radially coherent GAM" and discussed in the previous section has been repeated for the "radially varying" one, observed in the TCV discharge \#49032. This discharge features a ramp-up of the edge value of the safety factor and, as already discussed, beyond a given q edge value the observed axisymmetric oscillations lose their radial coherence, acquiring a spatially inhomogeneous character. The safety factor profile, as well as the measured temperature and density profiles, used for carrying out the simulations are plotted in Figure 13. Comparing with the profiles shown in Figure 2, besides the difference in
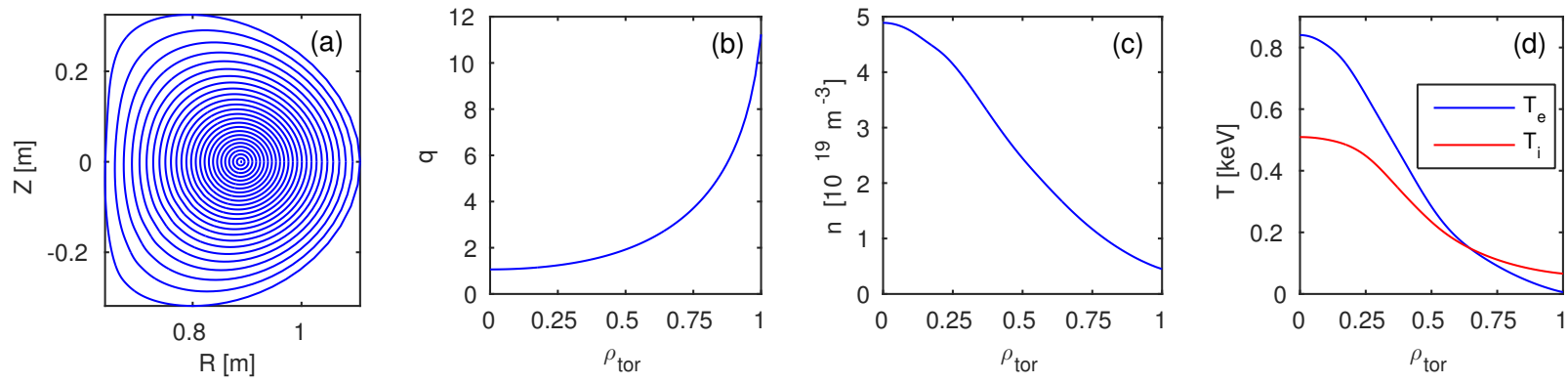

Figure 13: (a) Reconstructed magnetic equilibrium (shown are contours of constant poloidal flux), (b) safety factor $q$ profile, (c) electron density $n_{e}$ and $(d)$ electron and ion temperature profiles as a function of $\rho_{t o r}\left(T_{e}\right.$, in blue, and $T_{i}$, in red, respectively). Profiles measured for TCV discharge \# 49032 at time t=0.6s presenting a "radially varying GAM".

the safety factor profile, one observes that the electron density is significantly larger at all radial locations, and more than a factor of two in the core. At the same time the electron temperature is lower by up to $40 \%$ in the core. 
The ion temperature profile is also different, but with smaller relative variations. As a result, the ions are hotter than the electrons at almost all locations in the outer half of the plasma minor radius, with a ratio $T_{i} / T_{e}$ exceeding two as one approaches the LCFS. These differences in the profiles strongly impact also the logarithmic gradients (not shown here), which are significantly larger in the "radially-varying GAM" conditions compared to the "coherent" case, especially for the electron temperature and density. Even though impurities are not accounted for in the simulations, we observe also a large difference in the carbon concentration, significantly smaller in this discharge as reflected by the value of the effective charge $Z_{\text {eff }}=1.54$ instead of $Z_{\text {eff }}=3.4$ in discharge \#45886.

\section{B. Local simulation results}

Similarly to what was discussed in Section IV B, local simulations at different radial locations are carried out as a first step. Again, we look at the positions $\rho_{t o r}=0.6,0.7$ and 0.85. Experimental plasma parameters used for the simulations are listed in Table III. As already pointed out, the plasma conditions are significantly different compared

\begin{tabular}{lccc}
\hline \hline & $\rho_{\text {tor }}=0.6$ & $\rho_{\text {tor }}=0.7$ & $\rho_{\text {tor }}=0.85$ \\
\hline \hline$R_{0} / L_{T_{e}}$ & 12.36 & 13.27 & 21.62 \\
$R_{0} / L_{T_{i}}$ & 7.94 & 6.90 & 5.43 \\
$R_{0} / L_{n}$ & 8.18 & 9.32 & 10.61 \\
$T_{i} / T_{e}$ & 1.01 & 1.21 & 2.13 \\
$q_{0}$ & 2.44 & 3.20 & 5.15 \\
$\hat{s}$ & 1.53 & 2.01 & 3.08 \\
$\beta$ & $0.6310^{-3}$ & $0.2910^{-3}$ & $0.6910^{-4}$ \\
$\nu_{e i}$ & 1.35 & 2.36 & 7.92 \\
\hline \hline
\end{tabular}

Table III: Local parameters used for carrying out flux-tube simulations investigating the radially dispersive GAM conditions of TCV discharge \#49032.

to the ones considered in the previous section and summarized in Table I. The lower electron temperature and higher density translate into a larger collisionality, by almost a factor 20-30 everywhere. As will be further discussed when presenting global results, the different temperature results also in a different value of $\rho^{*}$, potentially leading to a different magnitude of global effects. Looking first at the electron parameters, logarithmic temperature gradients are at all positions larger for this discharge in comparison to \#45886 (see Table I), by approximately 30 to $40 \%$. Much larger is however the logarithmic density gradient, which at $\rho_{t o r}=0.6$ is almost six times the one considered when simulating the "radially coherent GAM". A somewhat smaller ratio is observed at $\rho_{\text {tor }}=0.7$, further reduced to $\sim 15 \%$ at $\rho_{\text {tor }}=0.85$. As for the main ion parameters, once more we observe a value of $R / L_{T_{i}}$ nearly twice as large everywhere.

All these large disparities make the two considered experimental conditions significantly different, thus any dissimilarity in the results cannot be attributed solely to the different value of the safety factor, as variations of other parameters can also play a relevant, if not major, role.

Linear growth-rates and real frequencies as a function of the $k_{y} \rho_{s}$ wave number are depicted in Figure 14 . For this particular parameter set, defining the kind of linear modes at play appears a less obvious exercise than in the previously analyzed discharge.

We observe how the frequency of the most unstable mode $\omega_{r}$, for all positions and independently from the inclusion of collisions, continuously increases from negative (i.e. the electron diamagnetic drift direction) to positive (the ion diamagnetic one) for the $k_{y} \rho_{s} \lesssim 1$ modes. For $k_{y} \rho_{s} \gtrsim 1$, a positive frequency branch follows at $\rho_{\text {tor }}=0.6$ and 0.7 , while at $\rho_{\text {tor }}=0.85$ the most unstable mode returns to $\omega_{r}<0$. One would be tempted to name these modes respectively TEM and ITG, given also their ballooning nature. However, when looking at a quasi-linear estimate of the heat fluxes, the contribution to the ion and to the electron heat channels is of the same order of magnitude for all $k_{y}$ modes, suggesting a more complex nature of these modes. Furthermore, the low $k_{y}$ positive frequency branches are destabilized when the ion temperature gradient is reduced and their frequency becomes negative (e.g. for the $k_{y} \rho_{s}=0.5$ mode at $\rho_{\text {tor }}=0.6$ the frequency becomes negative for $\left.R_{0} / L_{T_{i}} \leq 6\right)$. The high $k_{y} \rho_{s}$ branch also remains unstable and with a positive frequency when the ion temperature gradient is set to zero, i.e. completely suppressing the drive for ITG turbulence. This behaviour is understood as a consequence of the particularly high density gradient. Indeed when lowering $R_{0} / L_{n}$ towards values similar to the ones used in section IV B, one obtains a more "standard" TEM-like linear behaviour, with modes possessing a negative $\omega_{r}$ and a quasi-linear heat flux mainly through the electron channel. We also find that a smaller density gradient increases the growth-rate for all $k_{y}$ modes analyzed 

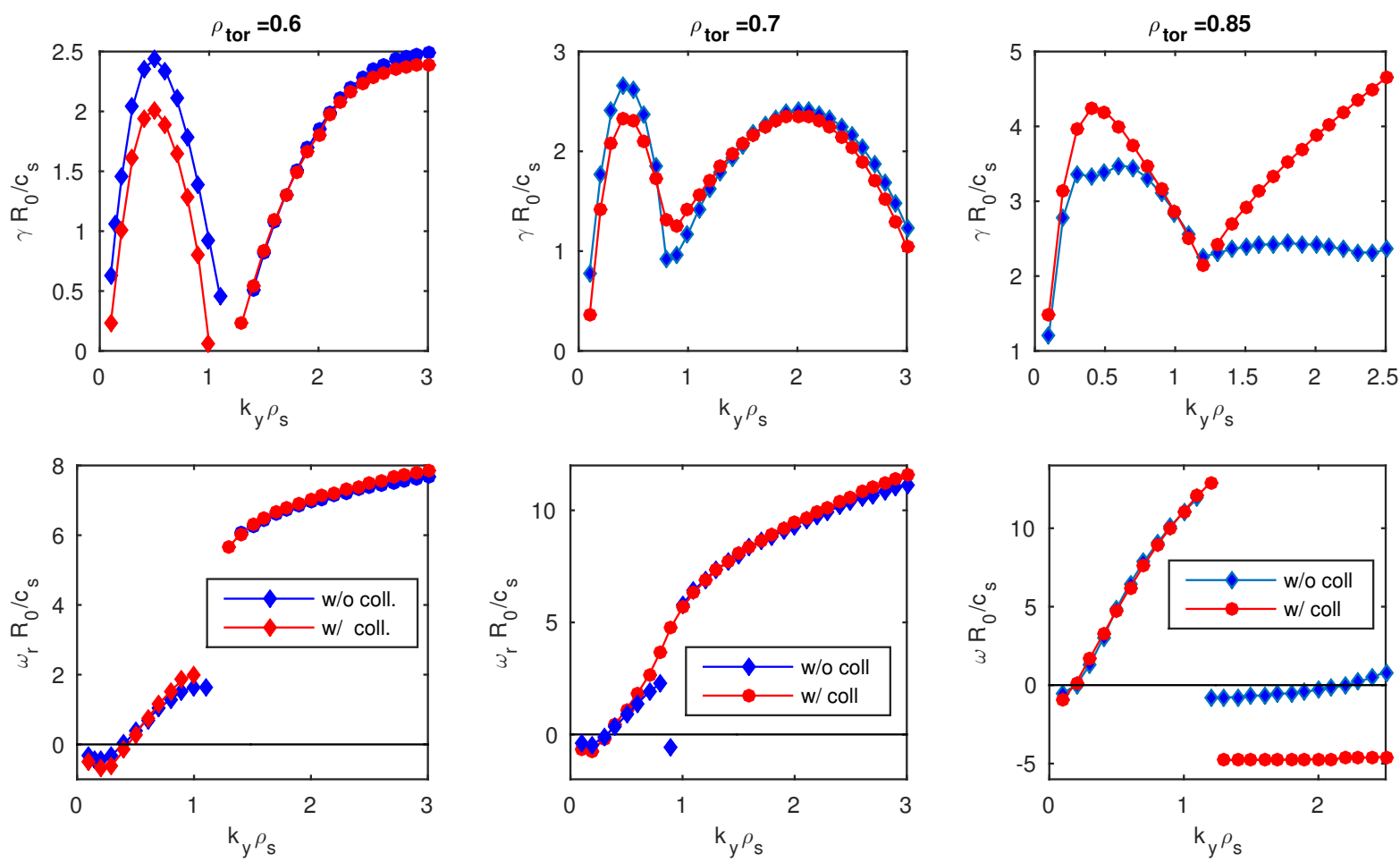

Figure 14: Growth rates $\gamma$ (top panels) and real frequencies $\omega_{r}$ (bottom panels) as a function of the wave number $k_{y} \rho_{s}$ evaluated at the radial locations $\rho_{\text {tor }}=0.6,0.7$ and 0.85 . Different markers are used to identify the different branches. Results obtained neglecting (resp. retaining) finite collisionality are depicted in blue (resp. red). Plasma parameters for the "radially dispersive GAM" scenario.

here. Finally, at $\rho_{t o r}=0.85$ we observe that the inclusion of collisions is destabilizing the modes, a signature typical of Resistive Ballooning Modes (RBM) [35]; RBMs are however expected to possess a negative frequency according to GENE conventions, thus once more clearly defining the nature of the low $k_{y}$ branch is non-trivial. Since part of this behaviour can potentially be caused by the extremely large density gradient, as well as be influenced by the heavy electrons assumption, we leave a detailed investigation of the nature of these modes to future work.

Nonlinear simulations have been carried out for the three different locations, with and without finite collisionality. The larger values of gradients and collisionality require a significantly smaller time step in comparison with the one used for the local simulations of the "radially coherent GAM". This is particularly relevant for the outermost location, and in order to reduce the computational effort associated to these simulations somewhat shorter statistics have been acquired, typically up to $100 R_{0} / c_{s}$. Such statistics are nonetheless sufficient to characterize the axisymmetric oscillations mode. The transport level obtained from these runs is, as expected, significantly larger than the experimental one. This will be discussed in more details in the next section when comparing with local simulations.

The temporal Fourier analysis of the simulated $\mathbf{E} \times \mathbf{B}$ velocity associated with the zonal flows has been repeated for this new set of runs. For simplicity we show only the radially averaged power spectral density in Figure 15. In all simulations a clear peak can be seen; the corresponding frequencies have been summarized in Table IV. As already observed when discussing the "radially coherent GAM" simulations, nonlinear oscillations have a frequency below the linear estimates, with a difference that is in this case approximately $10 \%$ for the two innermost positions and around $30 \%$ at $\rho_{\text {tor }}=0.85$. Collisions are once again found to affect the results by lowering the dominant frequency. This effect is more pronounced as one compares innermost to outermost positions.

\section{Global simulation results}

Following the same approach as in the "global GAM" scenario, the global simulation covers the outer half of the plasma, namely the region $0.5 \leq \rho_{\text {tor }} \leq 0.9$. The reference value of $\rho^{*}=\rho_{s} / a$ at the center of the simulations domain $\left(\rho_{\text {tor }}=0.7\right)$ is $\rho^{*} \simeq 1 / 293$ compared to $\rho^{*} \simeq 1 / 122$ in the coherent GAM case. The simulation radial domain is thus $L_{x} \simeq 135 \rho_{s}$. In order to use a similar radial resolution as the one adopted for the previous global run, we therefore needed in this case $n_{x}=416$ radial grid-points, which makes the simulation computationally more expensive. To 

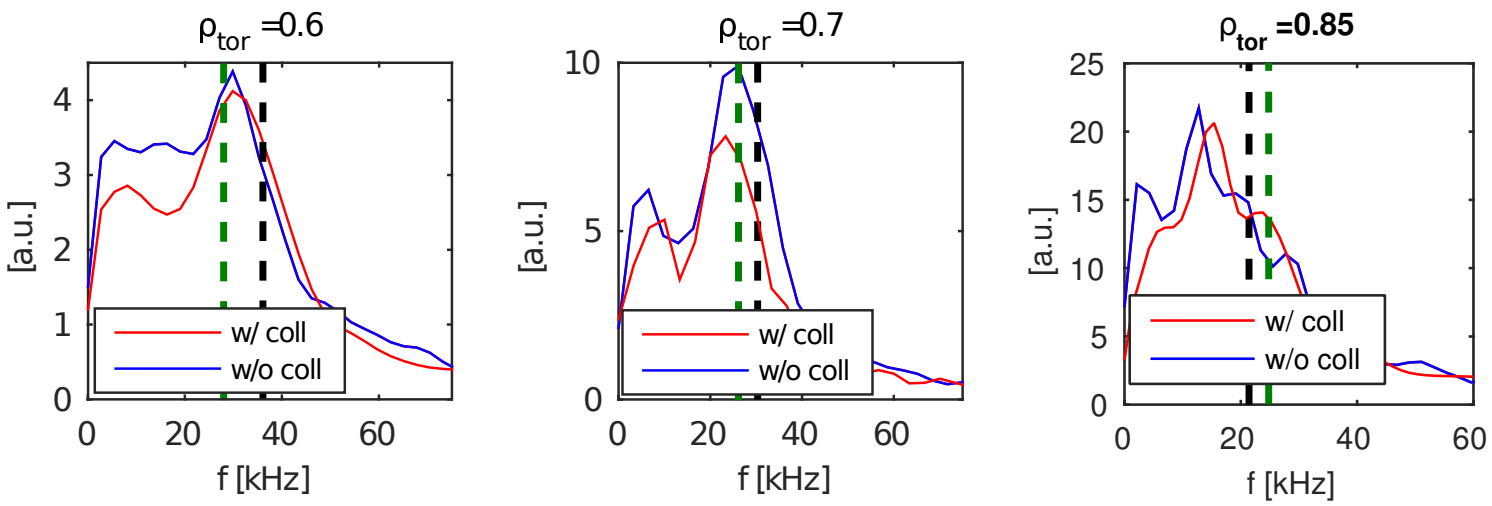

Figure 15: Same as Figure 6 but showing the results obtained modeling the conditions for the "radially varying GAM".

\begin{tabular}{lccc}
\hline \hline & $\rho_{\text {tor }}=0.6$ & $\rho_{\text {tor }}=0.7$ & $\rho_{\text {tor }}=0.85$ \\
\hline \hline $\mathrm{f}_{\text {lin, GAM }}[\mathrm{kHz}]$ & 36.0 & 29.2 & 21.4 \\
$\mathrm{f}[\mathrm{kHz}], \mathrm{w} / \mathrm{o}$ coll. & 32.5 & 26.0 & 12.7 \\
$\mathrm{f}[\mathrm{kHz}], \mathrm{w} /$ coll. & 32.5 & 22.8 & 16.8 \\
\hline \hline
\end{tabular}

Table IV: Dominant frequency, in $\mathrm{kHz}$, of axisymmetric oscillations obtained from analyzing the $\mathbf{E} \times \mathbf{B}$ velocity in nonlinear local simulations and the linear estimate obtained from the Rosenbluth-Hinton test. Runs considered parameters of the"radially varying GAM" case.

reduce the cost of the run only 32 toroidal mode numbers are considered here, while the same number of points, $n_{z}=32$, are used for the parallel direction. The same grids have been used in velocity space $\left(L_{v_{\|}} \times L_{\mu}=4.14 \times 18\right.$ and $n_{v_{\|}} \times n_{\mu}=110 \times 48$ points).

The resulting heat fluxes, together with the estimates obtained from local simulations, are shown in Figure 16. We observe that, as in the comparison presented in Figure 8, global simulations still significantly overestimate the
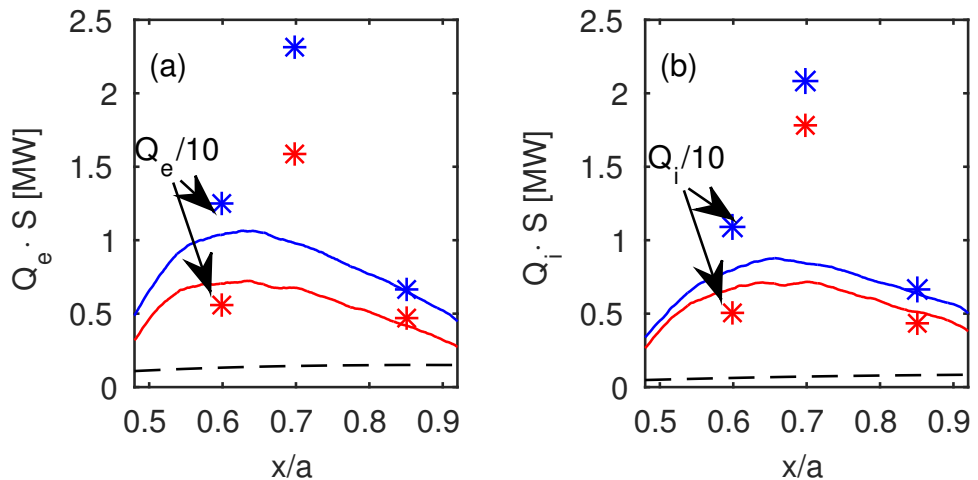

Figure 16: Global simulation results for "dispersive GAM" case. Total heat power in MW $Q \cdot S$ as a function of radius for (a) electrons and $(b)$ ions. Results obtained neglecting (resp. including) collisionality are shown in blue (resp. red). Flux-tube estimates are indicated with stars. In order to fit within the axis limit, local results obtained at $\rho_{\text {tor }}=0.5$ have been divided by 10 .

experimentally measured transport level, by at least a factor of 5 for the innermost locations, where simulation present the maximum heat transport. At these locations, flux-tube estimates are off by a factor of $\sim 10$ compared to the experiments. The heat transport obtained from flux-tube runs is thus again much larger than the global estimates, with the exception of the location $\rho_{\text {tor }}=0.85$, where local and global estimates coincide for both ion and electron channels. This is understood as a consequence of the very small local value of $\rho^{*}$ at this location $\left(\rho^{*}=1 / 461\right)$ such that global effects are expected to play a negligible role.

The temporal evolution of the simulated $\mathbf{E} \times \mathbf{B}$ velocity associated with the zonal flows is shown in Figure 17, while 
we plot in Figure 18 the corresponding Fourier time analysis. Inspecting the contours of the zonal $\mathbf{E} \times \mathbf{B}$ velocity, one
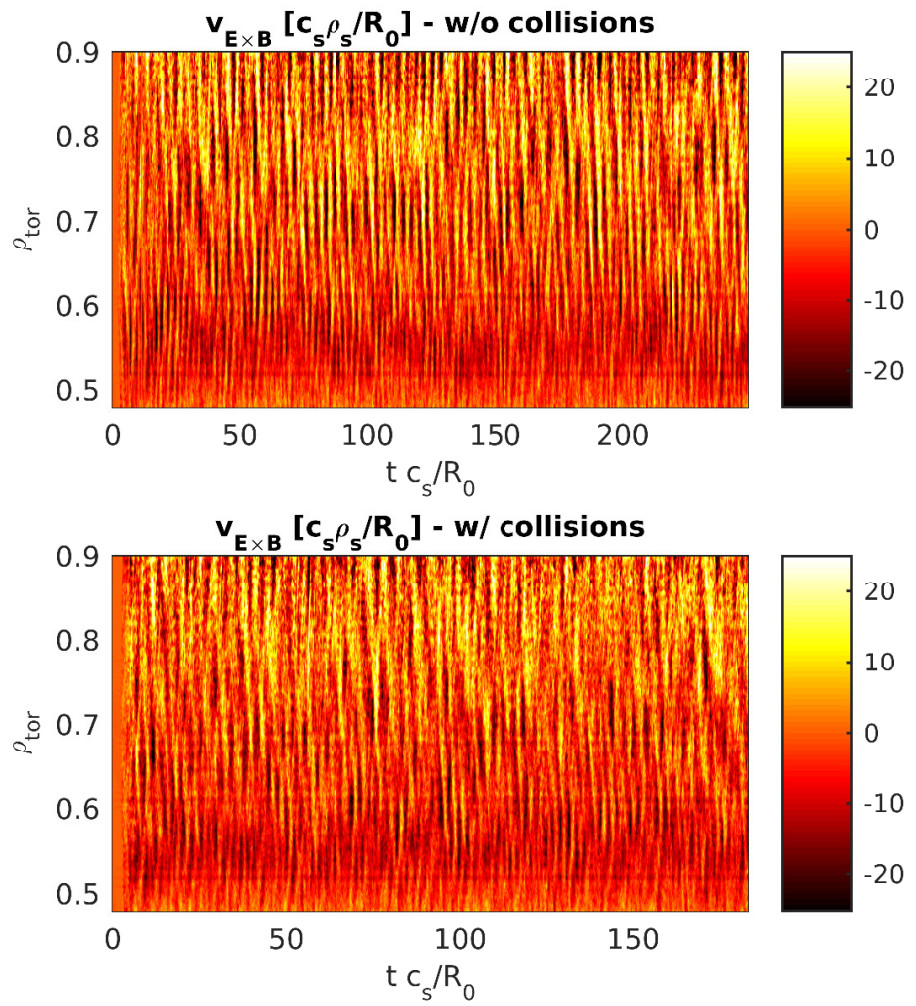

Figure 17: Same simulation as Fig.16. Evolution of the $\mathbf{E} \times \mathbf{B}$ velocity obtained from collisionless (top panel) and collisional simulation(bottom panel).
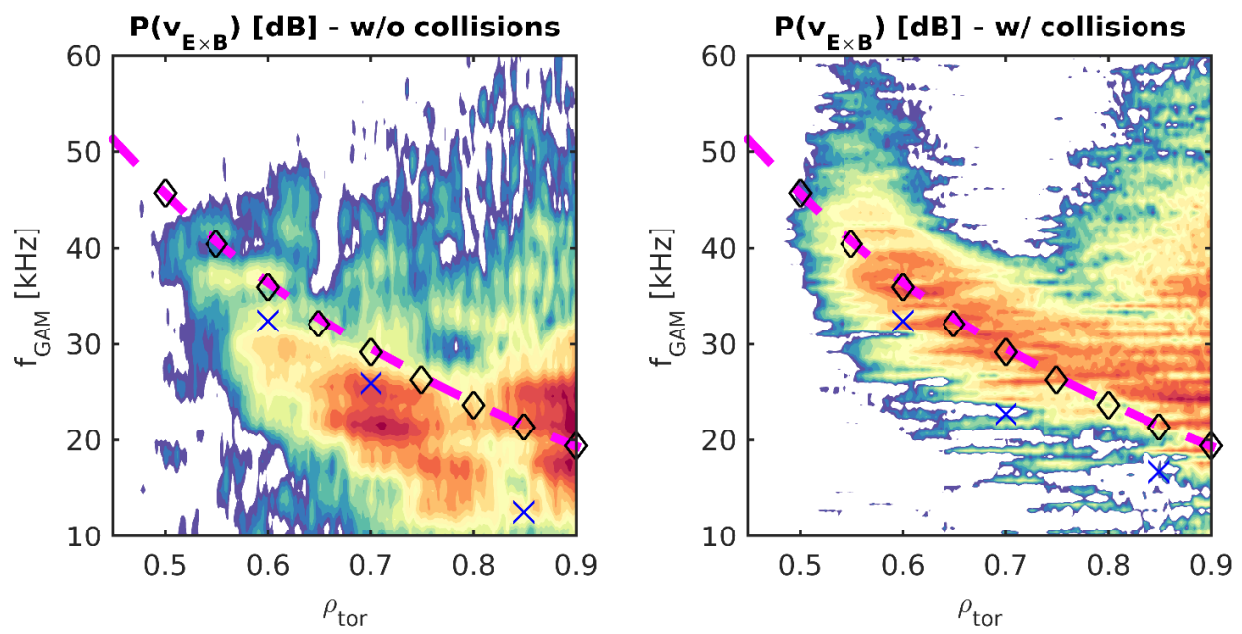

Figure 18: Same simulation as Fig.16. Power spectra density of the $\mathbf{E} \times \mathbf{B}$ velocity for the (left) collisionless and (right) collisional simulations, $\mathrm{dB}$ scale with the same color coding for the two cases. The magenta dashed line indicates the analytic linear GAM frequency estimate (Eq. (3), obtained from Ref. [5]), while black diamonds correspond to the frequency simulated in flux-tube via the Rosenbluth-Hinton test. Nonlinear local results discussed in the previous section are reported with blue crosses.

notes, as was already the case in the "global GAM" case (see Figure 10), the presence of oscillations that extend over most of the radial domain being simulated. The Fourier analysis however shows a very different behaviour. Contrary to the "global GAM" scenario, in this case we cannot identify the presence of a single or few localized oscillations that dominate the power spectra, but we rather observe contributions from different frequencies at different radial 
locations. In other words, we observe an axisymmetric oscillation that is radially dispersive and extends over almost all the outer half of the plasma minor radius. This is in agreement with what is experimentally measured.

A major role appears to be played by collisionality. The dispersive nature of the mode can be already observed in the collisionless run, where we find that the whole region $0.6 \leq \rho_{\text {tor }} \leq 0.8$ is characterized by oscillations with a frequency that varies with radius. It is however when we include collisions in our simulation that we obtain the clearest picture of radially dispersive axisymmetric oscillations, with a nearly uniform amplitude as a function of the radial position. The frequency that we measure in our simulations appears to follow the local linear GAM frequency (dashed lines in Figure 18). We nonetheless remark that when collisions are neglected, simulations provide a frequency that is downshifted with respect to the corresponding linear locate estimate, as already noted when discussing the "global GAM" scenario. The downshift appears in this case to be maximum at $\rho_{\text {tor }}=0.8$, while looking at even larger radii we observe a broader frequency spectrum. On the other hand, when collisions are included, the frequency reduction is much smaller and the nonlinear frequency is very close to the linear GAM frequency at each radial location. A lower nonlinear frequency with respect to the linear one is observed also in the local runs, between $10 \%$ and $20 \%$, and regardless of including or not collisions in the simulation model.

\section{DISCUSSION}

The experimental plasma conditions between the two discharges discussed in the previous sections are too disparate to attribute the different properties of the oscillations solely to the effect of the safety factor profile. Changes in temperature and density profiles lead to significant variations of all plasma parameters, in particular of the gradients, which drive the turbulence, but also of collisionality and of the finite machine size parameter $\rho^{*}$. Since all these effects can actively affect turbulence and in turn the behavior of $n=0$ oscillations, scans over these parameters should be performed in order to measure their actual role. Such an exercise is computationally very expensive (the simulations presented in this work have required approximately 10M CPU-hrs), in particular investigating the effect of the finite machine size because of its obvious impact on the resolution requirements.

We have started to pursue this investigation by performing two additional simulations where the temperature and density profile of the "radially coherent GAM" have been used with the magnetic geometry of the "radially varying" case, and vice-versa. Even though we have showed that collisionality appears to be one of the key ingredients for reproducing the correct behaviour of axisymmetric modes, it has been neglected in these last runs. The main motivation was to lower the computational cost of the simulations, still capturing the effect of the safety factor profile, as suggested by the experiments. The Fourier analysis of the $\mathbf{E} \times \mathbf{B}$ velocity is depicted in Figure 19 . These plots
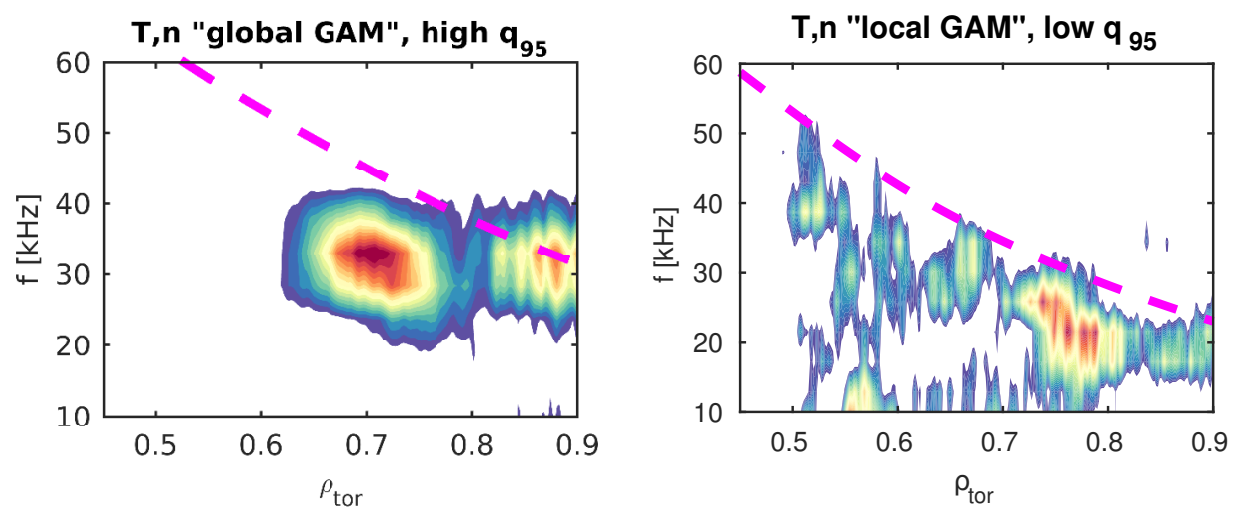

Figure 19: Power spectra density of the $\mathbf{E} \times \mathbf{B}$ velocity. Results shown on the left have been obtained considering the temperature and density profiles of the "radially global GAM" experimental conditions and the magnetic geometry of the "radially coherent" (large edge safety factor). The ones on the right show the opposite combination: $T, n$ of the of the "radially coherent" and geometry of the "radially global GAM" with low edge safety factor. The magenta dashed line indicates the analytic linear GAM frequency estimate provided in Ref. [5].

should be compared with the left panels of Figures 11 and 18. Looking first at the simulations carried out with the profiles of the "radially coherent GAM", we observe that even when the edge safety factor is increased the mode is found to maintain its radially global nature, see left plot of Fig 19 . The $\mathbf{E} \times \mathbf{B}$ velocity spectra are dominated by a single oscillation at $\sim 33 \mathrm{kHz}$, extending from $\rho_{\text {tor }}=0.65$ to the plasma edge and peaking at $\rho_{\text {tor }} \simeq 0.7$, which is somewhat inwards compared to the results obtained with the experimental $q$ profile. When looking at simulation results from the run considering temperature and density profiles of the radially-varying TCV conditions with the 
low edge safety factor we observe a richer frequency spectra, i.e. with multiple frequency peaks at different radii. The spectra in this latter case appear to be radially dispersive, though not as clearly as in Figure 18, with a peak at $0.7<\rho_{\text {tor }}<0.8$. Based on these results, we conclude that the safety factor is likely not to be the main cause for the regime transition, which appears instead to be rather related to the temperature and density profiles. This is contrary to previous conjectures, but has also been confirmed by more recent TCV observations [16].

One last point worth discussing concerns the nature of these fluctuations. In Figure 20 we plot the spatial and temporal evolution of the heat flux (for both ion and electron channels), the particle flux and of the $\mathbf{E} \times \mathbf{B}$ velocity. The results refer to the "radially coherent" case (same simulation as in Figure 8), but similar plots can be obtained for all simulations previously discussed. One observes that the flux profiles are characterized by ballistically propagating
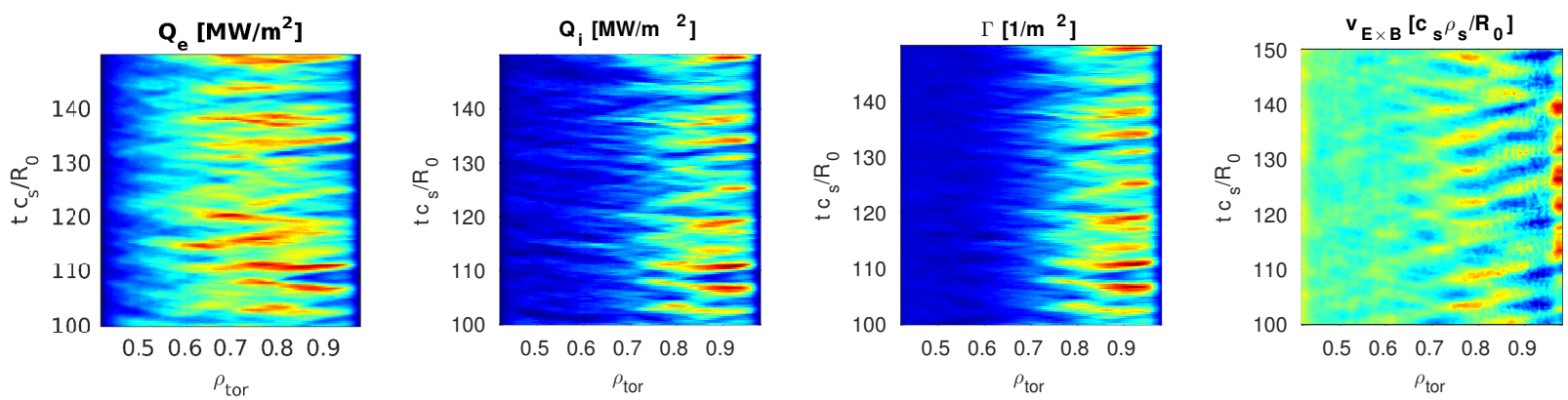

Figure 20: Same simulation as in Figure 8. From left to right, electron heat flux $Q_{e}$, ion heat flux $Q_{i}$, particle flux $\Gamma$ and $\mathbf{E} \times \mathbf{B}$ velocity. Simulation corresponding to the "radially coherent" GAM scenario.

structures, so-called avalanches $[22,36]$. While for the electron heat flux these structures extend over almost the whole radial domain, they are much more localized to the edge region for both the ion heat flux $Q_{i}$ and the particle flux $\Gamma$. Comparing with the $\mathbf{E} \times \mathbf{B}$ velocity (last panel of Fig. 20), some qualitative similarities can be observed regarding location and frequency of axisymmetric oscillations and transport avalanches, suggesting an interplay between the $\mathrm{n}=0$ mode (which itself does not drive any finite flux) and avalanches. Similar observations of a relation between axisymmetric oscillations and modulation of fluxes can be made also when analyzing flux-tube simulation results, as already noted in Ref. [36]. A nonlinear origin of the "radially global" GAMs has also been recently suggested in Ref. [37].

Possible experimental evidence of such a complex self-regulating mechanism has recently been obtained in diverted discharges in TCV, where oscillations in the particle flux at the same GAM frequency have been observed [16]. We however remark that since our simulations do not predict the correct transport level, this can only be confirmed by repeating simulations with the correct ion-to-electron mass ratio.

\section{CONCLUSIONS}

Nonlinear local and global gyrokinetic simulations have been performed with the aim of investigating the behaviour of axisymmetric $(n=0)$ fluctuations. Experimental TCV conditions have been considered as inputs, with the aim of qualitatively reproducing the actual observations. Two discharges involving two different safety factor profiles, one with moderate and one with large edge safety factor, have been investigated. Experimentally it is routinely observed that $n=0$ density fluctuations possess a global nature, i.e. a single frequency is measured over a large fraction of the plasma minor radius. One exception to this behaviour was observed during a safety factor ramp-up, where for large value of the edge safety factor these oscillations were found to become radially varying, thus the motivation for investigating the two different discharges. In order to reduce the computational effort, simulations were carried out with artificially heavy electrons $\left(m_{\mathrm{D}} / m_{e}=400\right)$. This choice is known to strongly affect the turbulent flux amplitudes, thus no particular effort was made to match the transport level between simulations and experiments, by varying e.g. the normalized profile gradient drives within their error bars.

Local simulations have been carried out at the radial locations $\rho_{\text {tor }}=0.6,0.7$ and 0.85 , whereas global runs have been used to simulate the outer half of the plasma minor radius. Two series of nonlinear simulations, either neglecting or including the effect of collisionality, have been performed for both local and global runs. Axisymmetric oscillations have been investigated by studying the temporal and spatial behaviour of the $\mathbf{E} \times \mathbf{B}$ velocity. In global simulations, oscillations of $v_{\mathbf{E} \times \mathbf{B}}$ are observed in the outer half of the plasma minor radius, mainly with an outward propagation, as shown in Figure 10. In local simulations however, periodic boundary conditions don't allow one to identify a preferred direction of propagation and a criss-cross pattern is then observed (Figure 5). An up-down asymmetry of 
the plasma is expected to induce a preferred direction of propagation also in the flux-tube simulations, [38]. The TCV discharges considered here are only weakly asymmetric, thus this effect is negligible here. In addition, aiming at understanding the role played by the safety factor, two extra simulations have been performed which interchange the experimental geometry and temperature and density profiles from the two experimental discharges with different magnetic geometries. For simplicity collisions have been neglected in these additional runs. The main findings can be summarized as follows:

(i) The "radially coherent GAM" discharge is characterized by unstable TEM and ETG modes. A more complex situation is observed for the "radially varying" case, where we observe also the presence of modes rotating in the ion diamagnetic direction. While this is the typical signature of ITG modes, we notice that these modes are able to drive, according to both quasi-linear and nonlinear estimates an almost equal heat flux through the ion and the electron channels. Furthermore the linear frequency remains positive even when zero ion temperature gradient is considered, and a standard TEM picture is recovered only when the density gradient is reduced.

(ii) The simulated fluxes strongly overestimate the actual measured transport level. This is understood mainly as a consequence of the heavy electron assumption. Strong global effects are observed on both discharges, with global simulations providing a significantly lower transport level compared to local results. It is however not obvious how to define an effective $\rho_{\text {eff }}^{*}$ in order to relate the magnitude of global effects to the actual temperature profile. The local and global results obtained modeling the "radially varying GAM" conditions can be reconciled by assuming that global effects are directly related to the local value of $\rho^{*}$. For the "radially coherent GAM" parameters, however, global effects provide the opposite trend. Local fluxes more strongly overestimate the global ones at the outermost radial positions.

(iii) In agreement with the experimental observations, when modelling the "radially coherent GAM" a well-defined frequency peak in the radially-averaged $\mathbf{E} \times \mathbf{B}$ power spectra is seen, while when the "radially varying GAM" conditions are modeled, $\mathrm{n}=0$ oscillations appear instead to be radially varying, with similar contribution from different radial locations. A good qualitative agreement with experimental measurements is obtained for both the magnitude of the frequency and the spatial location of these oscillations. Contrary to the experiments we however observe the existence of other axisymmetric components with lower amplitude and typically at a higher frequency. Finally, the nonlinear frequency of the $n=0$ mode is found in both local and global results to be significantly lower than the linear GAM frequency, with the exception of the "radially dispersive GAM" conditions, where the global nonlinear results seem to agree with local linear estimates.

(iv) The safety factor is observed to cause changes in the axisymmetric fluctuations, but it appears that the radial extent of the mode is not significantly affected by modifying the safety factor profile. The origin of the regime transition therefore has to be found in the different temperature and density profiles, either directly through the different local gradients driving the turbulence, or indirectly i.e. via the effect of collisionality or finite machine size.

(v) Similarities between the behavior of axisymmetric oscillations and transport avalanches are observed, in agreement with recent experimental observations. This suggests a complex interplay between $n=0$ and $n \neq 0$ fluctuations and questions the interpretation of the experimentally measured frequency as the nonlinear GAM frequency in favour of a characteristic avalanche frequency.

Further investigations are required to complement the present study, addressing also the impact of other paramters not accounted for here e.g., the effect of the plasma shape or the uncertainty in the temperaure and density profiles. In particular, given the non-linear nature of the axisymmetric fluctuations, reproducing the experimental amplitude levels appears to be an essential prerequisite before investigating their dynamics and the relation between linear GAMs, transport avalanches and turbulence self-organization. High realism global simulations, considering e.g. the realistic ion-to-electron mass ratio, are thus expected to be necessary. Such an effort is currently ongoing.

\section{ACKNOWLEDGEMENTS}

We wish to thank A.Biancalani for useful discussions. This work was supported by a grant from the Swiss National Supercomputing Centre (CSCS) under projects ID s617 and s704.Part of the computations were carried out using the HELIOS supercomputer system at the Computational Simulation Centre of International Fusion Energy Research Centre (IFERC-CSC), Aomori, Japan. This work was performed partly in the framework of the Helmholtz Virtual Institute on Plasma Dynamical Processes and Turbulence Studies using Advanced Microwave Diagnostics, and was also supported in part by the Swiss National Science Foundation. This work has been carried out within the framework 
of the EUROfusion Consortium and has received funding from the Euratom research and training programme 20142018 under grant agreement No 633053. The views and opinions expressed herein do not necessarily reflect those of the European Commission.

[1] P. H. Diamond, S.-I. Itoh, K. Itoh, and T. S. Hahm, Plasma Physics and Controlled Fusion 47, R35 (2005).

[2] K. Itoh, S.-I. Itoh, P. H. Diamond, A. Fujisawa, M. Yagi, T. Watari, Y. Nagashima, and A. Fukuyama, Plasma and Fusion Research 1, 037 (2006).

[3] Z. Lin, T. S. Hahm, W. W. Lee, W. M. Tang, and R. B. White, Science 281, 1835 (1998).

[4] N. Winsor, J. L. Johnson, and J. M. Dawson, Physics of Fluids 11 (1968).

[5] Z. Gao, Physics of Plasmas 17, 092503 (2010).

[6] C. Wahlbergh and J. P. Graves, submitted to Plasma Physics and Controlled Fusion (2016).

[7] W. Guo, S. Wang, and J. Li, Physics of Plasmas 17, 112510 (2010).

[8] K. DeMeijere, An experimental study of plasma fluctuations in the TCV and TEXTOR tokamaks, Ph.D. thesis, EPFL Lausanne (2013).

[9] G. D. Conway and the ASDEX Upgrade Team, Plasma Physics and Controlled Fusion 50, 085005 (2008).

[10] G. R. McKee, D. K. Gupta, R. J. Fonck, D. J. Schlossberg, M. W. Shafer, and P. Gohil, Plasma Physics and Controlled Fusion 48, S123 (2006).

[11] A. Fujisawa, Nuclear Fusion 49, 013001 (2009).

[12] L. Vermare, P. Hennequin, O. Gürcan, and the Tore Supra Team, Nuclear Fusion 52, 063008 (2012).

[13] C. A. de Meijere, S. Coda, Z. Huang, L. Vermare, T. Vernay, V. Vuille, S. Brunner, J. Dominski, P. Hennequin, A. KrämerFlecken, G. Merlo, L. Porte, and L. Villard, Plasma Physics and Controlled Fusion 56 (2014).

[14] Z. Huang, S. Coda, G. Merlo, C. de Meijere, J. Graves, S. Brunner, M. Fontana, L. Porte, L. Vermare, L. Villard, C. Wahlberg, J. Dominski, P. Hennequin, and A. Krämer-Flecken, in EFTSOMP (2015).

[15] S. Coda, Z. Huang, G. Merlo, L. Porte, S. Brunner, M. Fontana, and L. Villard, in US-EU Joint Transport Task Force Workshop (2011).

[16] Z. Huang, S. Coda, G. Merlo, S. Brunner, L. Villard, B. Labit, and C. Theiler, (2017), submitted to Plasma Physics and Controlled Fusion.

[17] K. Itoh, S.-I. Itoh, P. H. Diamond, A. Fujisawa, M. Yagi, T. Watari, Y. Nagashima, and A. Fukuyama, Plasma and Fusion Research 1, 037 (2006).

[18] Z. Gao, K. Itoh, H. Sanuki, and J. Q. Dong, Physics of Plasmas 15, 072511 (2008).

[19] G. Wang, W. A. Peebles, T. L. Rhodes, M. E. Austin, Z. Yan, G. R. McKee, R. J. L. Haye, K. H. Burrell, E. J. Doyle, J. C. Hillesheim, M. J. Lanctot, R. Nazikian, C. C. Petty, L. Schmitz, S. Smith, E. J. Strait, M. V. Zeeland, and L. Zeng, Physics of Plasmas 20, 092501 (2013).

[20] T. Ido, Y. Miura, K. Kamiya, Y. Hamada, K. Hoshino, A. Fujisawa, K. Itoh, S.-I. Itoh, A. Nishizawa, H. Ogawa, Y. Kusama, and J.-M. group, Plasma Physics and Controlled Fusion 48, S41 (2006).

[21] A. Melnikov, L. Eliseev, S. Perfilov, S. Lysenko, R. Shurygin, V. Zenin, S. Grashin, L. Krupnik, A. Kozachek, R. Solomatin, A. Elfimov, A. Smolyakov, M. Ufimtsev, and T. H. Team, Nuclear Fusion 55, 063001.

[22] B. F. McMillan, S. Jolliet, T. M. Tran, L. Villard, A. Bottino, and P. Angelino, Physics of Plasmas 16, 022310 (2009).

[23] Z. Huang, Ph.D. thesis, EPFL Lausanne (2017).

[24] F. Jenko, W. Dorland, M. Kotschenreuther, and B. N. Rogers, Physics of Plasmas 7, 1904 (2000).

[25] T. Görler, X. Lapillonne, S. Brunner, T. Dannert, F. Jenko, F. Merz, and D. Told, Journal of Computational Physics 230, 7053 (2011).

[26] See http://genecode.org/ for a description of the GENE code.

[27] X. Lapillonne, Local and global eulerian gyrokinetic simulations of microturbulence in realistic geometry with applications to the TCV tokamak, Ph.D. thesis, EPFL Lausanne (2010).

[28] G. Merlo, S. Brunner, O. Sauter, Y. Camenen, T. Görler, F. Jenko, A. Marinoni, D. Told, and L. Villard, Plasma Physics and Controlled Fusion 57, 054010 (2015).

[29] H. Lütjens, A. Bondeson, and O. Sauter, Computer Physics Communications 97, 219 (1996).

[30] B. F. McMillan, X. Lapillonne, S. Brunner, L. Villard, S. Jolliet, A. Bottino, T. Görler, and F. Jenko, Phys. Rev. Lett. 105, 155001 (2010).

[31] P. Welch, IEEE Transactions on Audio and Electroacoustics 15, 70 (1967).

[32] M. N. Rosenbluth and F. L. Hinton, Phys. Rev. Lett. 80, 724 (1998).

[33] J. Lang, S. E. Parker, and Y. Chen, Physics of Plasmas 15, 055907 (2008).

[34] D. R. Ernst, J. Lang, W. M. Nevins, M. Hoffman, Y. Chen, W. Dorland, and S. Parker, Physics of Plasmas 16, 055906 (2009).

[35] C. Bourdelle, X. Garbet, R. Singh, and L. Schmitz, Plasma Physics and Controlled Fusion 54, 115003 (2012).

[36] T. Görler, X. Lapillonne, S. Brunner, J. Chowdhury, T. Dannert, F. Jenko, B. F. McMillan, F. Merz, D. Told, and L. Villard, Journal of Physics: Conference Series 260, 012011 (2010).

[37] I. Novikau, A. Biancalani, A. Bottino, G. D. Conway, Ö. D. Gürcan, P. Manz, P. Morel, E. Poli, A. Di Siena, and the ASDEX Upgrade Team, ArXiv e-prints (2017), arXiv:1709.01818[physics.plasm-ph] . 
[38] R. Hager, Radial propagation of geodesic acoustic modes, Ph.D. thesis, Technische Universität München (2011). 